OPEN ACCESS

Edited by: Xiyun Deng,

Hunan Normal University, China

Reviewed by: Islam M. Miligy,

University of Nottingham, United Kingdom Petra Tesarova,

Charles University, Czechia

${ }^{*}$ Correspondence:

Eddy S. Yang

shyang@uabmc.edu

Specialty section:

This article was submitted to

Breast Cancer,

a section of the journal

Frontiers in Oncology

Received: 30 April 2021 Accepted: 23 August 2021 Published: 24 September 2021

Citation:

Clark CA and Yang ES (2021) Harnessing DNA Repair Defects to Augment Immune-Based Therapies in

Triple-Negative Breast Cancer.

Front. Oncol. 11:703802. doi: 10.3389/fonc.2021.703802

\section{Harnessing DNA Repair Defects to Augment Immune-Based Therapies in Triple-Negative Breast Cancer}

\author{
Curtis A. Clark ${ }^{1}$ and Eddy S. Yang ${ }^{1,2,3 *}$ \\ 1 Department of Radiation Oncology, University of Alabama at Birmingham (UAB) School of Medicine, Birmingham, \\ AL, United States, 2 O'Neal Comprehensive Cancer Center, University of Alabama at Birmingham (UAB) School of Medicine, \\ Birmingham, AL, United States, ${ }^{3}$ Hugh Kaul Precision Medicine Institute, University of Alabama at Birmingham (UAB) School \\ of Medicine, Birmingham, AL, United States
}

Triple-negative breast cancer (TNBC) has poor prognosis with limited treatment options, with little therapeutic progress made during the past several decades. DNA damage response (DDR) associated therapies, including radiation and inhibitors of DDR, demonstrate potential efficacy against TNBC, especially under the guidance of genomic subtype-directed treatment. The tumor immune microenvironment also contributes greatly to TNBC malignancy and response to conventional and targeted therapies. Immunotherapy represents a developing trend in targeted therapies directed against TNBC and strategies combining immunotherapy and modulators of the DDR pathways are being pursued. There is increasing understanding of the potential interplay between DDR pathways and immune-associated signaling. As such, the question of how we treat TNBC regarding novel immuno-molecular strategies is continually evolving. In this review, we explore the current and upcoming treatment options of TNBC in the context of DNA repair mechanisms and immune-based therapies, with a focus on implications of recent genomic analyses and clinical trial findings.

Keywords: TNBC, DNA repair, immunotherapy, PARP inhibition (PARPi), PD-1 - PD-L1 axis, DDR (DNA damage response), breast cancer

\section{INTRODUCTION}

Triple-negative breast cancer (TNBC) is defined by the absence of estrogen and progesterone receptors (ER and PR) and human epidermal growth factor receptor 2 (HER2). This aggressive variant, which accounts for $15-20 \%$ of all breast cancers (BC), exhibits a high propensity for early recurrence and metastasis $(1,2)$. Despite relatively better initial response rates to taxane- and anthracycline-based chemotherapy, durable responses are limited as a result of poorly differentiated tumors with higher rates of acquired resistance to systemic chemotherapy and radiotherapy as compared to other BC subtypes, with median overall survival in metastatic TNBC ranging from 1218 months $(1,3)$.

Understanding of specific heterogeneity in TNBC has served as the basis for certain targeted therapies based on particular molecular subtypes previously identified through genomic and transcriptomic profiling $(1,4)$ : The 1 ) basal-like (BL) subtype exhibits higher rates of BRCA1/2 mutations and expression of DNA damage response (DDR) genes; 2) mesenchymal-like (MES) 
subtype exhibits stem-like properties, and increased epithelialmesenchymal transition (EMT), phosphoinositide 3-kinase (PI3K), and Janus kinase (JAK) pathway activation; 3) immunomodulatory (IM) subtype is associated with increased immune checkpoint expression and tumor-infiltrating lymphocytes (TILs); and 4) luminal androgen receptor (LAR) subtype is associated with increased androgen receptor (AR) signaling. For instance, the BL subtype may be potentially more sensitive to alkylating agents, platinums, or poly (ADP-ribose) polymerase (PARP) inhibitors (PARPi's) as the result of higher rates of BRCA1/2 mutations and DDR deficiency, whereas the MES subtype may be sensitized to protein tyrosine kinase (PTK) and PI3K inhibition given increased activation of these pathways. Likewise, the IM subtype may have increased response to immunotherapy given increased TILs and expression of immune checkpoints, whereas the LAR subtype is potentially more sensitive to AR inhibitors given increased androgen-dependent metabolic activity in this molecular variant of TNBC. However, targeted therapies in TNBC have failed to achieve the remarkable efficacy as observed in other cancers $(1,5)$.

Pervasive therapeutic resistance in TNBC is another significant challenge, contributing to higher recurrence rates and decreased survival as compared to other BC subtypes (5). Therapeutic resistance in TNBC subtypes occurs through a variety of mechanisms. These include greater antioxidant and autophagy capacity resulting in resistance to radiation- or drug-induced oxidative stress, chemoresistance through upregulation of O-6methylguanine-DNA methyltransferase (MGMT)-associated activity and mismatch repair (MMR)-deficiency allowing for base mismatched DNA replication (6-8), increased Mcl-1 and Bcl-2-related antiapoptotic activity, and high degree of immunosuppression in part through recruitment of regulatory $\mathrm{T}$ cells (Tregs) (9), anti-inflammatory M2 macrophages (10), and increased immune checkpoint (e.g. PD-L1) expression (6, 11-13).

Nevertheless, based on the frequency of DDR deficiency in TNBC, investigation of novel strategies targeting DNA repair defects have generated hope for improved outcomes. PARPi's aimed at DDRdeficiency in TNBC have been approved for patients with metastatic HER2-negative BC with an inherited BRCA1 or BRCA2 mutation previously treated with chemotherapy (NCT02000622 using Olaparib), and those with deleterious or suspected deleterious germline BRCA-mutated HER2-negative, locally advanced, or metastatic BC (NCT01945775 using Talazoparib). However, these have restricted application and demonstrate modest albeit intriguing clinical benefit at present (14-16). A recent report also suggested benefit of PARPi in patients with metastatic breast cancer beyond germline BRCA1/2 mutations (NCT02032823 using Olaparib) (17).

Another promising therapy for TNBC exploits the immune system. Given the immunogenic characteristics of TNBC, immunotherapy represents a promising treatment strategy for this aggressive breast cancer with few efficacious systemic options at present. The most successful immunotherapeutic agents to date consist of immune checkpoint inhibitors (ICIs), which block immune co-inhibitory receptors, such as cytotoxic T-lymphocyte antigen 4 (CTLA-4) and programmed cell death protein 1 (PD-1), or associated ligands such as programmed cell death ligand 1 (PD-L1), to dis-inhibit TILs and permit tumor- specific cytotoxicity. However, highly immunosuppressive tumor microenvironment (TME) competes with ICI-enhanced antitumor immunity and significantly contribute to inconsistent clinical responses. Immunotherapies, particularly combination strategies, represent a refined approach to treating cancers with immune modulating DDR defects, high tumor mutational burden (TMB), and intact anti-tumor immunity, which are all characteristics frequently observed in TNBC. Tumors with intact interferon-gamma (IFN- $\gamma$ ) pathway signaling, robust TILs, increased immune co-inhibitory receptor expression, and high $\mathrm{TMB} /$ neoantigen expression have been shown to respond better to immune checkpoint inhibition than weakly immunogenic tumors with inadequately established anti-tumor immunity (18). As such, TNBC typically exhibits properties favorable to immunotherapy response, including increased TILs (19), which correlates with improved outcomes in early-stage TNBC (20), higher PD-L1 expression as compared to hormone receptor positive $\mathrm{BC}(12,13)$, and increased TMB giving rise to tumor neoantigen-specific $\mathrm{T}$ cells $(2,18,21,22)$. The PD-L1 mAb, Atezolizumab, is an FDA-approved ICI for patients with PD-L1 positive, unresectable, locally advanced, or metastatic TNBC (NCT02425891). However, ICI monotherapy efficacy is limited in TNBC, with response rates in the 5-25\% range (23), suggesting coexisting immunosuppressive or tumorigenic factors at play that overwhelm or subvert ICI-enhanced anti-tumor immunity. Thus, improved strategies that augment the immunotherapeutic potential of ICIs are needed.

Given the immunosuppressive phenotype associated with TNBC $(6,10,11,13)$, it is feasible that innate and acquired immune resistance mechanisms have in part curbed robust outcomes using various approved inhibitors in TNBC patients. Furthermore, DDR-targeting therapies have been shown to augment anti-tumor immunity as well as immune checkpoint signaling (24-27), potentially opening the door to combination immunotherapy in TNBC patients with DDR-deficiency and inadequate or exhausted TILs.

This review summarizes the promising role of DNA repair deficiency as a surrogate biomarker to guide the use of ICI therapy in TNBC, discusses underlying mechanisms that link DDR signaling to anti-tumor immunity, and outlines the emerging evidence describing the relationship and potential cooperative therapeutic potential between DDR-pathway targeting agents and immunotherapy.

\section{DNA DAMAGE REPAIR AND ASSOCIATED DEFECTS IN TNBC}

Cells routinely undergo DNA damage as the result of cytotoxic stress. In normal physiology, mechanisms of DNA damage detection and repair are critical to preserve genomic integrity and thwart malignancy when DNA damage exceeds the cellular repair threshold. DDR accomplishes this by arresting proliferation and facilitating removal of damaged cells through activation of senescence or apoptosis. As such, defects in DDR genes permit mutations and chromosome rearrangements 
advantageous for tumor initiation and progression. In TNBC, with alkylating chemotherapies and radiation as major components of therapy, aberrant DDR signaling represents a dominant mechanism of tumorigenesis and treatment resistance, while also yielding potential therapeutic synergies with platinum chemotherapies or targeted therapies. An overview of the DNA damage response and repair pathways is detailed below and shown in Figure 1.

\subsection{DNA Damage Response and Repair Pathways}

Depending on the mechanism of DNA damage and lesion formation, DDR is achieved by various pathways $(28,29)$. DNA single-strand break (SSB) damage is remedied by three main pathways: base excision repair (BER), nucleotide excision repair (NER), and mismatch-repair (MMR). More severe DNA double-strand breaks (DSBs) are restored by two additional pathways: homologous recombination (HR) and nonhomologous end joining $(\mathrm{NHEJ})(28,29)$. Ataxia telangiectasia mutated (ATM), ATM- and RAD3-related (ATR), and DNAdependent protein kinase (DNA-PK), in cooperation with many other mediators, act as core sensors that regulate DDR and coordinate DSB signaling. ATM and ATR protein kinases, operating together via downstream targets Checkpoint Kinase 1 (CHK1) and Checkpoint Kinase 2 (CHK2), respectively, play a vital role in DDR signaling by maintaining replication fork stability and the regulation of cell cycle control checkpoints (30). Additionally, DNA-PK activity is required for NHEJ, and a WEE1 nuclear kinase regulates mitotic entry and nucleotide reservoirs during DNA damage response $(30,31)$. Loss of function mutations in crucial genes involved in DDR, such as BRCA1/2, BRD4, PTEN or TP53, are associated with cancerprone cellular behavior and malignant phenotypes. Consequently, failure in DDR results in impaired removal of genome mutations, accumulation of DNA damage and increases the risk of oncogenesis (32). In reflexive response to DDR deficiency, tumor cells activate alternate DDR pathways, thereby counteracting sensitivity to genomic insult by

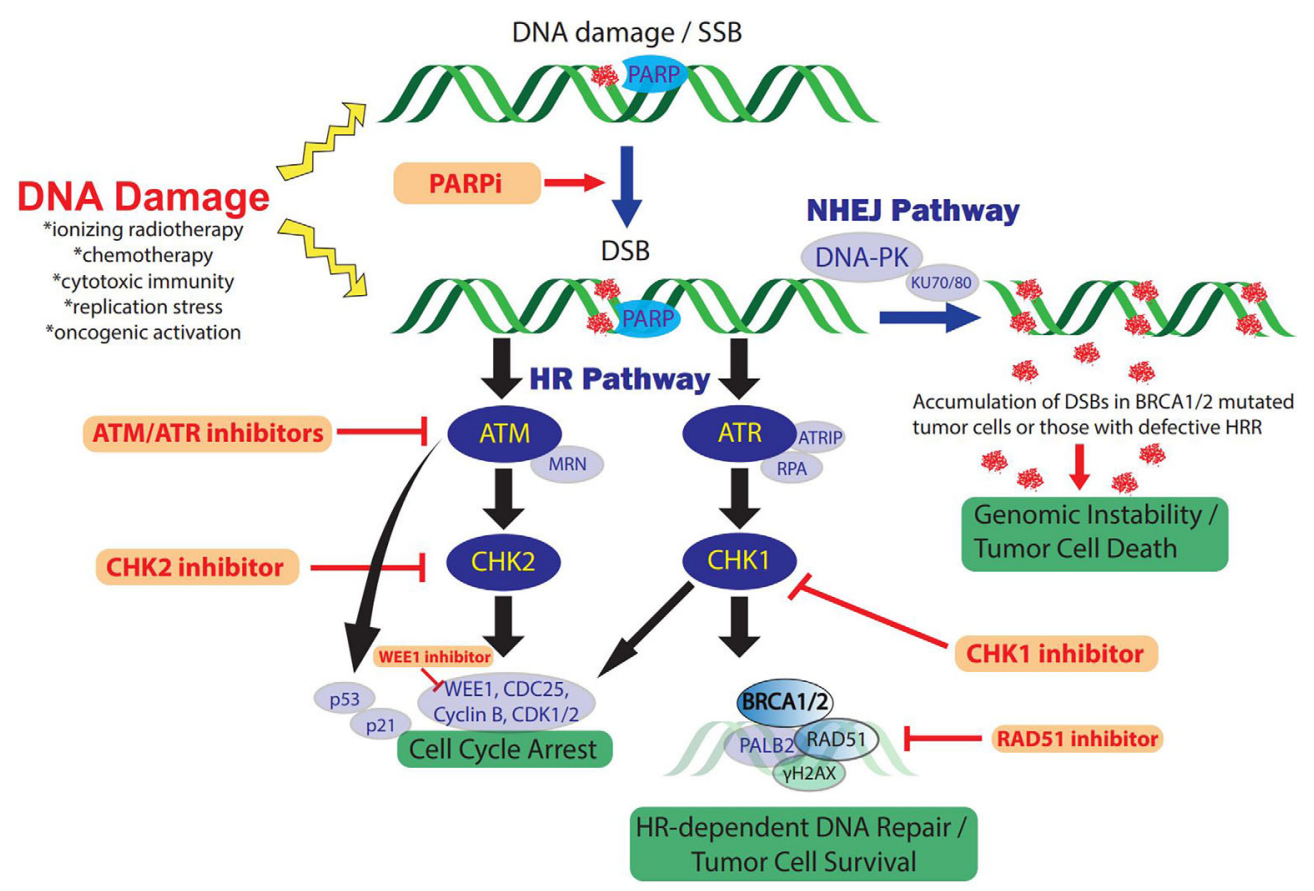

FIGURE 1 | The DDR and therapeutic strategies in TNBC. DNA-damaging therapies or endogenous replication dysfunction result in SSBs and DSBs which activate the DDR and repair signaling pathways. Distinct DSB DDR signaling pathway initiation depends on the type of DNA damage and is mediated by three central DDR kinases: DNA-PK, ATM, and ATR. In addition, PARP enzymes play a key role in DDR and facilitate SSB repair efficiency and functions in DSB repair via HRR and NHEJ pathways. The ATM and ATR pathways cross-talk extensively and only key intersections are highlighted here for pragmatic purposes. ATM/CHK2 signaling induces cell cycle arrest, preventing cell cycle progression in tumor cells with DNA damage. In addition, ATR/CHK1/WEE1 signaling initiates DNA DSB repair by inducing checkpoints and activating key components of HRR, including BRCA1/2 activity. Alternatively, DSB repair occurs through NHEJ via DNA-PKCS recruitment. Inhibition of PARP to treat TNBC with defects in HRR such as BRCA1/2 mutations, induces DSBs from unrepaired SSBs via PARP trapping and collapsed replication forks. Accumulated PARPi-induced DNA damage cannot be effectively repaired due to the HRR deficiency, resulting in genomic instability and cell cycle arrest. In addition, loss of function or inhibitors (red) against other key mediators of HRR also constrain NHEJ dependence which can be overwhelmed in the setting of concomitant PARPi via accumulation of DSB and genomic instability. RAD51 inhibition also suppresses HRR and sensitizes TNBC to PARPi. ATM/ ATR/CHK1MEE1 inhibition increases DSBs and impairs cell cycle arrest checkpoints and DNA damage repair, ultimately resulting in tumor cell death. DDR, DNA damage response; SSB, single-strand breaks; DSB, double-stranded breaks; DNA-PK, DNA-dependent protein kinase; ATM, ataxia telangiectasia-mutated; ATR, ataxia telangiectasia and Rad3-related protein; NHEJ, non-homologous end-joining; HR(R), homologous recombination repair; PARP, poly (ADP-ribose) polymerase; PARPi, PARP inhibitor/inhibition; MRN complex, Mre11, Rad50, Nbs1; ATRIP, ATR-interacting protein; RPA, replication protein A. 
preventing lethal cytotoxic stress and perpetuating oncogenesis, which is altogether a problematic mechanism of resistance to DNA-damaging cancer treatments. As a result of tumor cells often harboring oncogenic defects in DDR pathways and therefore increased dependence on alternate DDR mechanisms to survive, there is increased susceptibility to DDR inhibition and subsequent accumulation of lethal levels of DNA damage as compared to normal cells (5). These DDR defects will cause accumulation of significant DNA alterations that not only can facilitate oncogenesis, but it is becoming ever more evident that these changes can modify the TME and inflammatory cascade (33).

Therapeutic targeting of DDR pathways in TNBC is therefore a promising strategy given the propensity for therapeutic resistance and DDR deficiency. Furthermore, increasing evidence demonstrates a link between DDR deficiency and activation of anti-tumor immunity, and we will discuss the potential for combined approaches targeting genomic and immunologic aspects of TNBC tumorigenesis later in this review.

\subsection{The Role of PARP in DNA Damage Repair}

DNA base damage, such as base loss or SSBs, results in BER. Poly (ADP-ribose) polymerases (PARP1/2) are important DNAdamage sensors and regulators of BER-mediated SSB repair as well as other DDR pathways (25). These enzymes bind via zinc finger domains to SSBs via co-factor nicotinamide $(\beta-N A D+)$ and catalyze the synthesis of PARP chains (auto-poly (ADPribosylation), resulting in activation of intracellular signaling pathways that enable chromatin remodeling and recruitment of DDR-related protein machinery, thereby preventing accumulation of SSBs (34-36). In the setting of a HR deficiency, PARP inhibition disrupts efficient DNA damage repair resulting in increased genomic instability, stalled replication fork extension and lethal DSBs.

\subsection{Synthetic Lethality and Clinical Utility of PARP Inhibitors in TNBC}

Clinical use of PARP inhibitors (PARPi's) is an important example of DDR-specific targeting of HR defective cancers (14, 37). PARP1 inhibition can cause the accumulation of SSBs and subsequent DSBs. HR is required for DSB repair, and HRdeficiency is a typical pathological feature of the BRCA1/2mutated tumor and enables enhanced response to PARP1 inhibition due to synthetic lethality. PARPi's in cells deficient in HR are unable to effectively undergo DDR, whereas PARPi is well-tolerated by normal cells. As such, this effect of PARPi is more likely observed in tumor cells with a BRCA-deficient background or tumors with underlying deficiencies in HR (38). Tumors cells with intact HR signaling can overcome PARP inhibition preferentially by HR rather than NHEJ (34), whereas cells with HR deficiency (HRD), including those with mutations in BRCA1/2, BRD4, and PTEN, demonstrate sensitivity to PARP inhibition resulting in cell death $(35,36$, 38). PARPi therefore represents a synthetic lethal therapeutic approach for the treatment of cancers with compromised ability to repair double-strand DNA breaks by HR, including those with defects in BRCA1/2 $(17,34,38)$. Numerous PARPi's have been developed, including Olaparib, Rucaparib, Niraparib, Talazoparib, and Veliparib, which are primarily applied in cancer patients with BRCA1/2 mutations (14, 16, 17, 39). Altogether these studies demonstrate that sensitivity of HRDTNBC tumor cells to DNA-damaging agents may be the direct result of associated defective DDR mechanisms.

Although the greatest efficacy of PARPi has been observed in tumors with BRCA1/2 mutations, consensus is that synthetic lethality insufficiently explains PARPi-related anti-tumor activity. For example, the degree of PARP catalytic inhibition is poorly correlated to PARPi-induced cell-killing in HRD cells (40). In addition, PARPi induces cytotoxicity to a greater extent than PARP depletion, suggesting associated mechanisms contribute to anti-tumor activity $(40,41)$. In addition, loss of other tumor suppressor DDR proteins, many of which are involved in HR, such as RAD51, ATR, ATM, CHK1, CHK2, and partner and localizer of BRCA2 (PALB2), also have been shown to permit sensitization to PARPi $(35,40)$. HRD has also been shown to regulate sensitivity to alkylating chemotherapy in some TNBC patients (42), whereas the ATR-CHK1 cascade may conversely regulate resistance to chemotherapy by preventing replication stress. Further emphasizing the role of these accessory molecules in preventing susceptibility to DNA repair targeting, it was reported that ATR inhibition was effective in sensitizing both HR-proficient and deficient TNBC cells to ionizing radiation therapy (43). These results suggested that PARPi might be a useful therapeutic strategy not only for the treatment of BRCAmutated tumors but also for the treatment of a wider range of nonBRCA-mutated tumors that are inherently HRD or 'BRCAness/ HRDness' $(15,34)$.

In the context of TNBC, there is a higher degree of 'BRCAness' as compared to other breast cancer subtypes $(1,5)$, As such, PARPi's have demonstrated the potential for increased therapeutic efficacy in TNBC patients with HRD/BRCAness, due to increased accumulation of DSBs and incidence of synthetic lethality $(35,36)$. Olaparib, an orally active PARPi, was the first to be shown to induce synthetic lethality in BRCA-deficient cells and exhibit potential clinical benefit in patients with TNBC having BRCA deficiency. At present, Olaparib and Talazoparib are FDAapproved as single-agent regimens for previously chemotherapytreated, HER2 negative, metastatic breast cancers with germline BRCA mutations, which primarily constitutes TNBC. In addition to exploiting BRCAness in TNBC, PARPi's have been shown to radiosensitize breast cancer cells through DDR inhibition, and clinical trials in breast cancer patients explore their potential to enhance the response of cancers to ionizing radiation (44).

Use of PARPi's in TNBC is supported by findings from the phase III OlympiAD trial of metastatic breast cancer (16), which demonstrated an approximately two-fold increase in response rate $(59.5 \%)$, increased median progression-free survival (PFS; 7.0 months), and less toxicity as compared to conventional chemotherapy in patients with metastatic HER2-negative breast cancer with germline BRCA1/2 mutations treated with Olaparib (NCT02000622). A phase I study of Talazoparib demonstrated promising efficacy and safety profiles in 
advanced cancers with deleterious BRCA1/2 mutations including breast cancer (NCT01945775). A phase III trial EMBRACA comparing Talazoparib versus physician's choice standard of care in metastatic TNBC revealed significant benefit of Talazoparib with better PFS and objective response rates (ORRs) (14, 39). Other PARPi's, including Veliparib, and Rucaparib, have been investigated in metastatic breast cancer. Trials of veliparib in combination with alkylating agents are currently underway for advanced or metastatic TNBC (45-47). In early TNBC, the phase III OlympiAD trial (NCT02032823) is currently ongoing to evaluate adjuvant Olaparib monotherapy after standard neoadjuvant therapy in high-risk TNBC with germline BRCA1/2 mutations. Another phase I trial of neoadjuvant monotherapy with the novel PARPi Niraparib is underway (NCT03329937). The phase II/III PARTNER trial of neoadjuvant Olaparib in combination with carboplatin followed by the standard chemotherapy is under investigation in patients with TNBC and/or germline BRCA mutations (NCT03150576). The I-SPY 2 trial, which evaluated neoadjuvant Veliparib and carboplatin in addition to the standard chemotherapy in patients with high-risk breast cancer and TNBC, demonstrated significant benefit from this combination therapy (pathologic complete response (pCR) rates: $52 \%$ vs 24\%) (48). In a recent biomarker analysis of the I-SPY2, a BRCA1ness gene signature was identified as a significant predictive biomarker of response to neoadjuvant combination Veliparib and carboplatin (49). Conversely, a phase II neoadjuvant trial in high-risk, residual TNBC after standard neoadjuvant chemotherapy failed to show a significant therapeutic benefit from the combination of low-dose Rucaparib and cisplatin compared with cisplatin alone; although the lack of benefit may be due to a therapeutically insufficient rucaparib dose (NCT01074970). Altogether, additional studies are required to elucidate the clinical benefit of PARPi addition to platinum-based chemotherapy in TNBC as platinum alone demonstrates efficacy either as monotherapy or in combination $(50,51)$. This also further emphasizes the need to identify additional therapies that sidestep resistance to therapeutic targeting of DDR deficiency.

Other strategies to exploit HR include inducing a synthetic lethality by generating a BRCAness phenotype. These promising preclinical studies include combinations with inhibitors of EGFR, PI3K, BET, and others (52-54). We recently reported promising results of a clinical trial with lapatinib and veliparib in non-BRCA1/2 mutated TNBC based on an induced DNA repair deficiency with EGFR inhibition (NCT02158507) (55).

\subsection{Role of MMR and NHEJ in TNBC}

In TNBC, defective MMR allows DNA replication with mismatched bases and facilitates resistance to anti-metabolites and alkylating agents. Whole-genome sequencing studies have shown that approximately $5-7 \%$ of TNBC patients are MMRdeficient $(6,8)$, as compared to approximately $2 \%$ in other breast cancers. Furthermore, MMR status corresponds to PD-L1 expression and $\mathrm{CD}^{+} \mathrm{T}$ cells in the TNBC TME versus poor correlation in other subsets of breast cancers. Altogether, these findings indicate the immunotherapeutic efficacy potential in TNBC with MMR deficiency (6). In the context of immunotherapy, MMR deficiency not only has the potential to elicit more tumor antigens and improved immune checkpoint inhibitor response (56). The TMB/neoantigen/IFN- $\gamma$ pathway is a well understood cancer pathway that results in PD-L1 upregulation, supported by the finding that even partial loss of MMR significantly correlates with increased PD-L1 expression suggesting a therapeutic vulnerability in HRD TNBC (6). Mounting evidence indicates that DDR defects are also important in driving sensitivity and response to ICI. Given that MMR deficient (dMMR) tumors harbor a large number of mutations, which are associated with high neoantigen load and T-cell infiltration, it is not surprising that dMMR tumors can respond well to immune checkpoint blockade. Indeed in many cancers, MMR deficiency predicts efficacy of anti-PD-L1 (Pembrolizumab), and microsatellite instability (MSI)/dMMR is a validated DDR defect biomarker for predicting response to ICI therapy (56). Furthermore, Pembrolizumab is FDA-approved for solid tumors based solely on the presence of MSI-status as a biomarker, irrespective of cancer type (56). Although MSI or dMMR rarely appears in breast cancer (57), as we will discuss further, the therapeutic potential in combining with immunestimulating DNA repair inhibitors remains intriguing.

The NHEJ signaling pathway is an important mediator of DSB repair. The Ku70-Ku80 heterodimer and DNA-dependent protein kinase catalytic subunit (DNA-PKcs) initiate NHEJ, and these complexes have been shown to be regulated by EGFR amplification and/or p53 mutation-induced overexpression of long non-coding RNA in the NHEJ pathway 1 (LINP1), resulting in NHEJ-mediated chemo- and radiation resistance $(58,59)$. Doxycycline, an FDA-approved agent that can inhibit DNA-PK, has been shown to reduce DNA-PKcs expression and sensitize breast cancer cells to radiation (60). Although more investigation is necessary, these findings suggest that targeting of NHEJrelated mediators may be useful in TNBC, particularly those with EGFR, p53 and/or DDR-associated mutations resistant to DNA-damaging agents.

\subsection{Role of Radiation Therapy in DNA Damage Signaling and Immune Strategies}

Most breast cancer patients receive ionizing radiotherapy (RT) as part of their treatment to improve locoregional control by inducing tumor cell death predominately through the generation of DSBs, which in turn can elicit either protective anti-tumor immune responses or immunosuppression (61). Unfortunately, positive immune effects of radiation are often insufficient to shift the balance of the immunosuppressive TME to achieve tumor rejection, especially in the absence of targeted immunotherapy. Combining immune checkpoint blockade with radiotherapy has thus emerged as an exciting dual modality treatment approach for a myriad of cancer types, although clinical outcomes are highly variable.

\subsubsection{Impact of Radiation Therapy on Anti-Tumor Immunity and Immunosuppression}

RT-enhanced tumor immunogenicity can occur through multiple mechanisms, including increased antigen availability, inflammatory cell infiltration into tumors, and increased priming 
and exposure of phagocytic and cytotoxic cells to tumorassociated antigens (62). Specifically, RT can up-regulate FAS (death receptor) and MHC class I on tumor cell surfaces, alter the repertoire of peptides presented by $\mathrm{MHC}$, cause translocation of calreticulin to tumor cell surfaces resulting in enhanced antigen uptake by antigen presenting cells, and induce release of HMGB1 from dying tumor cells. These actions induced by RT can result in dendritic cell maturation and chemokine and cytokine secretion that promotes TIL trafficking $(62,63)$. Furthermore, RT-induced DSBs and subsequent ATM activation has been shown to regulate pattern recognition receptors that activate interferon and innate immune system signaling (64, 65). Local and systemic immune effects include RT-induced alteration of chemokine signaling, cell trafficking, and secondary immune system activation via dendritic cell cross-presentation of tumorderived antigens to $\mathrm{T}$ cells $(63,66)$.

The link between radiation and both local and systemic antitumor immune effects has been investigated in many preclinical and clinical studies $(61,63,65)$. It has been reported that immunerelated therapeutic effects of locally ablative RT require intact immunity, type I interferon production and infiltration of $\mathrm{CD}^{+}$ $\mathrm{T}$ cells (67), highlighting the importance of functional anti-tumor immunity in the current era of radio-immunotherapy. However, RT and the resultant tumor cell death can also potentiate immunosuppressive TMEs, as studies have shown that radiation can induce lymphopenia, immune dysfunction through release of immunosuppressive cytokines (TGF- $\beta$, IL-10) and chemokines, and induction of immunosuppressive immune cells including myeloidderived suppressor cells (MDSCs), M2 tumor-associated macrophages (TAMs), T regulatory cells (Tregs), which can all result in immune escape and tumor progression $(62,66)$.

Importantly, radiation can further induce immunosuppression and adaptive immune resistance via upregulation of checkpoint pathways, including PD-L1 expression on the tumor cell surface $(65,68)$. Although the neoantigen-T cell activation-IFN- $\gamma$-STAT1/ 3-IRF1 pathway of PD-L1 induction has historically been viewed as the chief mediator of this adaptive immune resistance, recent work has implicated DNA damage and repair signaling in the regulation of tumor $\mathrm{PD}-\mathrm{L} 1$, including through radiation-mediated DSBs and cytosolic DNA sensing. DNA damage dependent PD-L1 expression is upregulated by ATM/ATR/CHK1 kinase activities and the cyclic-GMP-AMP ((cGAMP) synthase (cGAS))/ stimulator of interferon genes (STING)-dependent pathway. Altogether, tumor cell PD-L1 expression is controlled by the STAT-IRF pathway which is regulated by distinct DNA damage mechanisms: 1) DSB-induced ATM/ATR/CHK1 kinase activities, 2) DDR deficiency/high $\mathrm{MSI} /$ increased TMB resulting in neoantigen-induced $\mathrm{T}$ cell activation and IFN- $\gamma$ production, and 3) cytosolic DNA fragments that induce the cGAS/STING pathway resulting in type I interferon activity (68).

RT induced PD-L1 expression via activation of the cytosolic DNA sensing cGAS/STING pathway represents a novel mechanism of adaptive immune resistance. The cGAS/STING, with subsequent type I interferon production, is a fundamental immunostimulatory pathway in antimicrobial innate immunity (64), and has been found to mediate the TME and immune milieu, including immune surveillance, dendritic cell function and $\mathrm{CD}^{+} \mathrm{T}$ cell function (69). Interestingly, STING-activity is also upregulated in the setting of DDR deficiencies including BRCA1/2 and ATM mutant tumor cells (69). This STINGdependent interferon signaling can initially facilitate immune activation; however chronic STING pathway activation and/or IFN- $\gamma$ signaling can ultimately lead to T cell exhaustion via PDL1-dependent resistance to anti-tumor immunity (70), potentiating cancer immune escape.

RT-induced DSBs and subsequent ATM/ATR/CHK1 kinase activities have also been implicated in upregulation of tumor PDL1 expression through direct STAT1/3-IRF1 activation (26, 66, 68), independent of neoantigen production. Consistent with this, $\mathrm{Ku}$ or BRCA2 defects were found to augment RT-induced PD-L1 expression $(26,68)$, and ATR inhibition reduced upregulation of PD-L1 following RT. Interestingly, ATR inhibition potentiated $\mathrm{CD}^{+} \mathrm{T}$ cell activity and reduced RT-induced $\mathrm{T}$ cell exhaustion (71). Furthermore, RT-induced interferon signaling has been shown to be dependent on cGAS/STING pathway activation (65). This evidence suggests a novel PD-L1-dependent, immunosuppressive consequence of DNA damaging therapies (e.g., chemotherapy, RT, DDR inhibitors). In relation to immune-activating properties of RT, the disadvantageous PDL1 induction following RT represents a therapeutic opportunity with combination ICI therapy that would result in more durable clinical responses.

\subsubsection{Clinical Application of Radio-Immunotherapy Combinations in TNBC}

Observations in patients receiving ICI and RT have demonstrated the potential for improved clinical responses in various primary and metastatic malignancies, and numerous clinical trials are underway investigating potential synergy. Clinical trials evaluating patients with metastatic cancer have established that RT combined with ICI is safe and well-tolerated, and can potentially halt tumor growth by stimulating anti-tumor immunity $(61,66)$. In TNBC, a phase II trial evaluated PD-L1 inhibition (Pembrolizumab) plus RT in patients with metastatic TNBC patients who were unselected for PD-L1 expression. In this study, the ORR for the entire cohort was $17.6 \%$ (3 of 17 patients; 95\% CI: $4.7 \%-44.2 \%$ ), with 3 complete responses of tumors outside of the irradiated portal (72). The context dependence of the robust synergistic effects of RT and ICI are potentially consistent with fluctuating immune-tolerance and suppression mechanisms, particularly in the locally advanced or metastatic setting. This altogether highlights the need for larger clinical trials assessing predictive biomarkers and investigation of additional targeted strategies. For instance, the phase I RADIOPARP trial is investigating PARP1 inhibitors (Olaparib) in combination with RT in the setting of advanced or metastatic TNBCs (73). Neoadjuvant Veliparib combined with RT is under exploration in a phase I study for node-positive, residual BC following neoadjuvant chemotherapy (NCT01618357). These and additional studies are needed to optimize radiotherapy modulation of DDR-dependent immune augmentation and anti-tumor immunity in the context of ICIs. 


\section{CANCER IMMUNOLOGY IN TNBC}

Immune evasion is a hallmark of cancer that is the result of a complex TME consisting of stroma, myeloid and lymphoid immune cells, dysregulated lymphovascular networks. The interaction of these components often plays roles in tumorigenesis, tumor heterogeneity, and adaptive and therapeutic resistance. Central to immune-mediated tumor rejection are TILs, a heterogeneous population that contributes to competing innate and adaptive anti-tumor and immunosuppressive effects. TILs, including $\mathrm{CD}^{+} \mathrm{T}$ and $\mathrm{NK}$ cells that are central to anti-tumor immunity in breast cancer, have prognostic significance even in systemically untreated early TNBC, suggesting that the presence of TILs may delineate candidates most likely to benefit from adjuvant chemotherapy or immunotherapy (74).

The TME of TNBC is often abundant in TILs because of inherent genomic instability and high mutational burden. As the result of these genetic and epigenetic aberrations, anti-tumor TILs engage in immune-mediated tumor cell killing and tumor cell immunoediting, often times resulting in subset(s) of immune resistant tumor cells $(2,75,76)$. In metastatic TNBC, response rate and overall survival after Atezolizumab significantly correlated with TIL levels (77). However, in early TNBC, retrospective studies demonstrated significantly worse survival outcomes in patients harboring high PD-L1 expression and a low number of TILs or a high ratio of PD-L1/CD8 expression (12, 78), suggesting that TIL alone is not indicative of the immune activity or suppression status. Consistent with this, immunologic signatures associated with higher mutational burden positively correlated with higher TILs and a more favorable prognosis (12), suggesting antigen-specific anti-tumor TILs likely play a significant role in coordinating the functional state of antitumor immunity and response to immunotherapy (12). Furthermore, TILs are shown to be a robust predictive biomarker of long-term survival in TNBC patients treated with neoadjuvant therapies and to facilitate improved response to cytotoxic agents (79-81). However, effective anti-tumor TIL activity is frequently hindered by immunosuppressive immune cells types such as regulatory Tregs and MDSCs, which are also typically found in higher concentrations in TNBCs. Nevertheless, compared with other BC subtypes, TNBC exhibits a higher degree of lymphocytic infiltration (19), and studies to date indicate that TILs are useful biomarkers and potential therapeutic targets in TNBC.

\subsection{Immune Co-Inhibitory Pathways in TNBC}

Upon activation, $\mathrm{T}$ cells begin to express co-inhibitory cell surface receptors that control T cell function, such as CTLA-4 and PD-1. The balance between co-stimulatory and coinhibitory signals is crucial for cytotoxic $\mathrm{T}$ cell activation and immunologic tolerance. Tumors can exploit this balance to escape $\mathrm{T}$ cell-mediated, tumor antigen-specific immunity. Importantly, therapeutically targeting these co-inhibitory pathways with immune checkpoint inhibitors (ICIs) is capable of unleashing anti-tumor activity $(78,82)$. In TNBC, immune coinhibitory signaling is often upregulated and is associated with immunosuppression, MMR-status and mutational burden, chemoresistance and overall poor prognosis $(6,11,12)$.

CTLA-4, an immune checkpoint constitutively expressed on Tregs and transiently upregulated on activated $\mathrm{T}$ cells, inhibits early $\mathrm{T}$ cell priming by antigen-presenting cells (APCs) in the lymph nodes (83). The expression of CTLA-4 on Treg cells competitively blocks the binding of $\mathrm{CD} 28$ to the $\mathrm{CD} 80 / 86$ proteins on APCs, thereby turning off $\mathrm{T}$ cell activation (82). CTLA-4 blockade has demonstrated efficacy in anti-tumor immune activity in some cancers by allowing tumor antigen-specific $\mathrm{T}$ cell stimulation. CTLA-4 ICI has demonstrated durable response in a small subset of patients with metastatic TNBC $(84,85)$, and CTLA-4 mAbs, including Ipilimumab and Tremelimumab, are being investigated with the PD-1-axis immunotherapies Durvalumab and Nivolumab, respectively, for TNBC.

PD-1, another immune checkpoint, is widely expressed on activated anti-tumor immune cells, including $\mathrm{T}$ and natural killer (NK) cells, and APCs, and yields inhibitory signals through binding of its two ligands, namely PD-L1 and PD-L2 (86). PDL1 is highly inducible and expressed on many cancers in response to anti-tumor immune activity and inhibits $\mathrm{PD}-1^{+}$ tumor antigen-specific $\mathrm{CD}^{+} \mathrm{T}$ cells (87), representing a key mechanism underlying cancer adaptive immune resistance. Correlation between TILs and PD-1/PD-L1 expression is well studied, as tumor-associated inflammation promotes adaptive upregulation of immunosuppressive PD-L1 expression in response to anti-tumor immune cell production of IFN- $\gamma$ and tumor cell STING pathway activation $(22,88)$. Blockade of PD$1 / \mathrm{PD}-\mathrm{L} 1$ interaction is capable of restoring $\mathrm{T}$ cell function and tumor elimination. However, in breast and other cancer cell types, meaningful response is inconsistent as a result of reduced or heterogeneous PD-L1 expression, immunosuppressive mechanisms, impaired immune cell function and trafficking of TILs $(87,89)$, resulting in paradoxical PD-L1 $1^{+}$"non-responders" and PD-L1 1 low/null "responders".

There is compelling evidence that resistance to DNA-damaging agents may play a meaningful role in immunotherapy outcomes. For example, defects in BRCA1/2 correlates to higher levels of PDL1 expression $(90,91)$. In addition to inactivation of PD- $1^{+}$antitumor immune cells, tumor PD-L1 also mediates diverse cellintrinsic functions that increase cancer virulence, including mTORC1 promotion and autophagy suppression (92-94), that can not only alter immune infiltrates and enable immune escape (94-98), but may also play a role in response to DNA-damaging therapies. Indeed, it has been shown that tumor-intrinsic PD-L1 can regulate IFN- $\boldsymbol{\gamma}$-induced apoptosis, DDR, RT and chemotherapy resistance, and effects on Ras/Mek/ERK, PI3K/ AKT, JAK/STAT (94, 99-101); which, altogether may create treatment-exploitable immune signaling effects.

The interaction of these pathways to modulate the immune system is depicted in Figure 2.

\subsection{Role of Immune Checkpoint Inhibition in TNBC}

ICIs, including monoclonal antibodies against $\mathrm{PD}-1$ (Pembrolizumab, Nivolumab), PD-L1 (Atezolizumab, Durvalumab, Avelumab), and CTLA-4 (Ipilimumab), have 


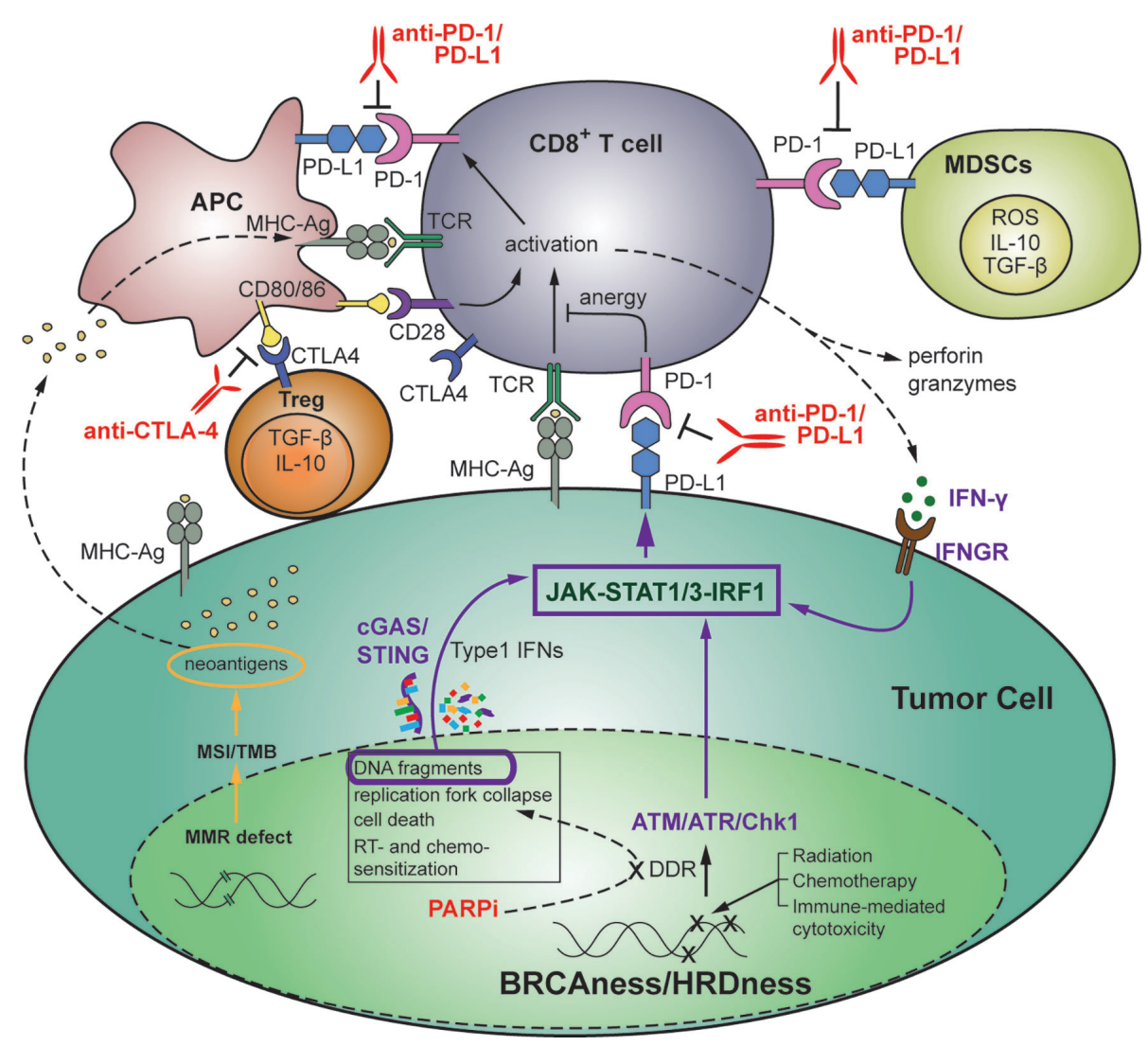

FIGURE 2 | Therapeutic strategies targeting the interplay between DDR and anti-tumor immunity in the setting of HR-deficient triple-negative breast cancer. DNA damage affects the balance between tumor progression and immune surveillance. Genomic stress induced by DNA-damaging treatments or by defects in DDR or MMR results in accumulation of chromosomal abnormalities, higher TMB, oncogene activation and tumorigenesis, as well as immune recognition, activation of immunostimulatory genes, and increased TILs including anti-tumor immune cells (CD8 ${ }^{+} \mathrm{T}$ cells, APCs, CD4 ${ }^{+} \mathrm{T}$ cells, and NK cells). Immunosuppressive immune cells such as CD4 ${ }^{+}$Tregs, MDSCs and M2 macrophages can also be increased. Targeting of SSB and DSB repair with inhibitors of DDR, including PARPi's, in the setting of TNBC with BRCA or HR-related mutations (BRCAness/HRDness) can, in addition to inducing synthetic lethality, increase generation of cytosolic DNA fragments. This results in activation of the immunomodulatory cGAS/STING pathway that promotes anti-tumor immunity through activation of T and NK cells, neoantigen recognition, and increased PD-L1 expression via the JAK-STAT1/3-IRF1 pathway. Anti-tumor immunity can further contribute to tumor PD-L1 expression via IFN- $\gamma$-dependent activation of IRF1. Tumor and immune cell expressed PD-L1 subsequently suppresses PD- ${ }^{+}$cytotoxic anti-tumor immune cells via inhibitory binding. Thus, DNA-damage induced anti-tumor immune response is often overwhelmed by coexisting immunosuppressive factors, and the balance in favor of antitumor immune rejection can be mediated by ICls such as anti-PD-1/PD-L1 and anti-CTLA-4 mAbs. APC, antigen presenting cells; ICI, immune checkpoint inhibitor; mAb, monoclonal antibody; MDSC, myeloid-derived suppressor cells; MMR, mismatch repair; NK, natural killer; TIL, tumor-infiltrating lymphocyte; TMB, tumor mutational burden; Treg, T regulatory cell.

generated durable responses across many tumor types (102). Clinical studies using PD-1/PD-L1 mAb therapies have demonstrated promise in patients with PD-L1 positive TNBC, and Atezolizumab is FDA-approved for patients with PD- $\mathrm{L1}^{+}$, unresectable, locally advanced, or metastatic TNBC (NCT02425891). Studies of Atezolizumab in advanced solid cancers, including heavily pretreated TNBC, demonstrate limited but impressive outcomes, as only $10 \%$ of patients experienced clinically meaningful response, with $100 \%$ survival rate at 2 years in these responders, and median PFS of PD-L1 ${ }^{+}$TNBC patients treated with Atezolizumab plus nab-PTX was significantly increased by $50 \%$ (7.5 months vs. 5 months) (103). KEYNOTE012 and KEYNOTE-086 studies demonstrated durable response with Pembrolizumab in approximately $20 \%$ of patients with metastatic TNBC $(104,105)$. Patients with positive PD-L1 expression treated with first-line Pembrolizumab showed a higher response rate than patients with any level of PD-L1 expression. Using Avelumab in patients with heavily pre-treated metastatic TNBC, the phase I JAVELIN trial demonstrated promising efficacy outcomes with a $31 \%$ control rate, and $\mathrm{PD}$ L1 expression correlated with response (106). Currently, a phase II trial of Pembrolizumab as monotherapy for BRCA-mutated breast cancer is underway (NCT03025035). These studies using PD-1axis inhibitors demonstrate therapeutic benefit in some patients, but future studies are required to address inconsistent responses, better define the therapeutic ceiling of ICIs in upfront treatment of early-stage TNBC, elucidate the role of targeted therapies in increasing therapeutic index of ICIs, and identify reliable biomarkers to guide the imperfect prognostic value of PDL1 expression. 
Based on the remarkably durable responses in a small subset of TNBC responders in ICI monotherapy studies, many studies using combination ICI with conventional therapies are currently ongoing and have shown early signs of benefit. Interim data from Impassion130 trial, using nab-paclitaxel in combination with Atezolizumab showed a 40\% ORR in metastatic TNBC, and early data suggests a clinically meaningful overall survival benefit in patients with PD-L1 immune cell-positive disease (NCT02425891). Trials investigating the combination of Eribulin and Pembrolizumab in heavily pretreated metastatic TNBC are ongoing, with interim analysis demonstrating a $41.2 \%$ ORR to first-line treatment and a 27.3\% ORR to later-line treatment (107). However, PD-L1 status failed to predict treatment response to either combination. These trials investigating ICI efficacy in heavy-treated TNBC patients altogether have highlighted the need for earlier intervention with ICI therapy in advanced or metastatic TNBC. KEYNOTE-355, a phase III trial evaluating the combination of Pembrolizumab plus conventional chemotherapy compared with chemotherapy alone as first-line treatment in metastatic TNBC is ongoing (NCT02819518). The combination of Durvalumab and nab-paclitaxel followed by dose-dense conventional chemotherapy as well as the combination of Avelumab and an antibody to the immune modulator, $41 \mathrm{BB}$, is under investigation in advanced solid tumors, including TNBC (NCT02489448).

In early TNBC, preliminary results from the neoadjuvant ISPY 2 trial demonstrated that pCR rates increased from $22.3 \%$ to $62.4 \%$ by adding neoadjuvant Pembrolizumab to paclitaxel followed by anthracycline-based chemotherapy, which represents an approximately $40 \%$ improvement in pCR compared with standard chemotherapy alone (108). The KEYNOTE-173 trial also showed a remarkably increased pCR rate from $60 \%$ to $90 \%$ in high-risk patients by combining Pembrolizumab with paclitaxel or conventional chemotherapy (109). In the adjuvant setting, the SWOG1418 phase III trial is evaluating adjuvant monotherapy with Pembrolizumab after neoadjuvant chemotherapy followed by curative surgery. Another phase III trial for high-risk patients with early TNBC is investigating the addition of Avelumab after standard curative treatment including adjuvant chemotherapy (NCT02926196).

Clinical success using immune checkpoint inhibitors has led to the identification of additional checkpoints that mediate tumor immunosuppression, such as the lymphocyte-activation gene 3 (LAG3), T cell immunoglobulin and mucin-domain 3 containing-3 (TIM3), Siglec-15, indoleamine 2, 3-dioxygenase 1 (IDO1), and glucocorticoid-induced tumor necrosis factor receptor (GITR). Targeted therapies for these are undergoing clinical trials in TNBC patients. For example, Siglec-15 is an immune checkpoint that inhibits antigen-specific $\mathrm{T}$ cell responses, and is expressed, independent of PD-L1 status, on both tumor and tumor-infiltrating myeloid cells (110), and a $\mathrm{mAb}$ for Siglec-15 is currently being evaluated in a phase I/II study for advanced or metastatic solid tumors (NCT03665285).

Although clinical trials for immunotherapy in breast cancer have not shown that same high efficacy as in other carcinomas, TNBC is likely to have increased benefit as compared to other types of breast cancer given high mutational load, DDR- deficiency and increased PD-L1 expression. This may be especially true in early-stage TNBC with potentially more favorable tumor immune microenvironments, as studies thus far have mostly evaluated immune checkpoint inhibitors in advanced staged TNBC. However, innate, and adaptive resistance to immunotherapy remains a challenge, and targeted therapies that synergize with the immune-activating potential of immune checkpoint inhibitors is a promising strategy to maximize immunotherapeutic potential in TNBC patients.

\section{DDR DEFICIENCY-ASSOCIATED ANTI-TUMOR IMMUNITY IN TNBC}

Recent work has highlighted the important interaction between genomic instability and the immunogenicity and activation of anti-tumor immunity (111). Highly mutated tumors often exhibit one or several mutations in key components of DDR or replicative pathways, including $\mathrm{MSH} 2$ for $\mathrm{MMR} / \mathrm{MSI}, \mathrm{BRCA} 1 / 2$ for $\mathrm{HR}$ and DNA polymerase epsilon (POLE) for DNA replication. Targeting of DSB repair proteins with DDR inhibitors has also been shown to increase the TMB (111). Likewise, DDR defects result in accumulation of chromosomal abnormalities, leading to higher TMB, oncogene activation and tumorigenesis $(112,113)$. However, this DDR-defect-dependent genomic instability and increased TMB can also result in immune recognition, activation of immunostimulatory genes, increased TIL, and anti-tumor immune production of IFN- $\gamma$ with resultant immunosuppressive tumor PD-L1 upregulation $(18,90$, $114,115)$. Similar effects can be observed as a result of genomic stress induced by DDR defects or DNA-damaging treatments, including RT, PARPi or platinum-based chemotherapies. This is due to generation of chromosomal fragments that stimulate the cytosolic sensing cGAS/STING pathway that promotes anti-tumor immunity through activation of $\mathrm{T}$ and NK cells, neoantigen recognition, and increased $\mathrm{PD}-\mathrm{L} 1$ expression, and this immune system stimulation is enhanced in the background of BRCAness/ HRDness $(24,27,90,116-118)$. It is also evident that in response to DNA damage, ATM/ATR/CHK1 kinase activity regulates the transition from DDR to immunostimulatory signaling directly through STAT1/3-IRF1-mediated transcription of PD-L1 (26). Thus, DDR signaling and DNA-damaging treatments result in robust immune modulation and significantly affect the balance between tumor progression and immune surveillance.

In support of the notion that tumor cells with extensive genomic instability orchestrate a high octane anti-tumor immune response that is smothered by coexisting immunosuppression, DNA damage and DDR-defects associated with increased TMB and neoantigen production correlate with STING-induced PD-L1 expression and improved ICI response (25). In some studies including in invasive breast carcinoma, defects in BER or BRCA1/2 were associated with increased neoantigen load, increased TILs, and elevated PD-L1 expression $(26,90,91,119)$, and a genome wide genetic screen identified BRCA2 inactivation as a mediator of cGAS/STINGinduced IFN response and pro-inflammatory cytokine production 
(116). Consistent with these findings, DDR deficient breast tumors exhibited increased immune infiltration. However, elevated PD-L1 expression was driven predominantly by cGAS/STING pathway activation as opposed to the canonical neoantigen/activated T cell/ IFN- $\gamma$ pathway of PD-L1 induction (69), which is significant given that STING activation mediated by DNA-damaging agents is implicated in response to ICI therapy. Although some studies report elevated TILs in BRCA1/2 mutant breast cancer $(21,24)$, a pooled analysis of five phase II studies showed that TIL density was not associated with HR defect or BRCA1/2 mutation in early stage patients with TNBC (20). It is therefore likely that neoantigenindependent mechanisms of immune augmentation are involved in TIL density and PD-L1 expression in DDR-deficient TNBC, which is compatible with numerous studies that have shown tumors with low TMB can also be sensitive to ICIs (22). Importantly, patients with BRCA1/2 and other HR-related gene deficiencies demonstrate higher response rates to ICI as compared to MMR deficient tumors despite relative lower TMB, corroborating the possibility of additional immunologic mechanisms related to DDR-deficiency $(22,120,121)$. Furthermore, the observation that HR intact tumors may also respond to the PARPi and ICI combination could perhaps be explained by the activation of the cGAS/STING and subsequent neoantigen-independent immune activation $(122,123)$.

Despite the significant clinical activity of PARPi in breast cancers harboring germline loss-of-function BRCA mutations $(14,16,39)$, the majority of patients treated with PARPi's alone do not significantly benefit (115). Unrepaired chromosomal damage following PARPi further promotes immune activation and adaptive upregulation PD-L1 expression via cGAS/STING pathway activation or ATM/ATR/CHK1 kinase activity (26, $119)$, which may result in immune escape and explain variable results. Altogether these studies support the hypothesis that use of PARPi together with ICI will retain immune activating consequences of DDR defect targeting while also preventing $\mathrm{T}$ cell inactivation.

In the setting of DDR deficiency, a consequence of tumor cell DNA damage and sustained inflammatory activity is recruitment and activation of immunosuppressive immune phenotypes as the result of chronic, low level, DNA damage, potentially resulting in cancer progression and immunotherapy resistance (117). It is proposed that PARPi may potentially shift to more substantial DDR-mediated cytotoxic anti-tumor immune milieu more favorable for ICI efficacy (124). In support, it is reported that PARPi efficacy is enhanced by $\mathrm{CD}^{+} \mathrm{T}$ cell activity via cross-talk with STING pathway activation in BRCA-deficient models of TNBC (123). Collectively, numerous studies indicate PARPidependent immunologic effects may prime a vigorous albeit imbalanced anti-tumor immune response and set the stage for improved ICI efficacy.

The combination of enhanced immune activation resulting from deficient DDR pathway signaling and the immunosuppressive consequences, including PD-L1 upregulation of unrepaired DNA damage via HR-deficiency and/or the use of DDR inhibitors such as PARPi, suggests potentially targetable immunological susceptibilities in TNBC patients (Figure 2). Tumor immune evasion mechanisms in response to genomic instability subvert immune-mediated elimination of DDR hindered cancers, serving as rationale for targeting the immunosuppressive arm of DDR signaling in response to DNA damaging therapies via ICI combinations. This approach may be highly lethal to immunogenic tumor cells with DDR defects and impinge upon these immunosuppressive mechanisms of therapeutic resistance $(24,68,71,125,126)$. TNBC often harnesses DDR defects, TMB load, and PD-L1 expression, and these characteristics have been found to be amongst the strongest predictors of response to ICI (18, $22,113)$.

\subsection{DDR Inhibitors and Immunotherapy in TNBC}

Therapeutic targeting of genomic instability through the use of DDR-inhibitors, including PARPi, have been shown to not only induce synthetic lethality in DDR-deficient tumor cells, but also to augment the tumor immune microenvironment through increased TMB and activation of immunostimulatory genes (21, $25,114)$. Accruing evidence supports the potential association between DDR defects and ICI efficacy. Interestingly, preclinical TNBC studies demonstrated PARPi-mediated PD-L1 upregulation with expected attenuation of anti-tumor immunity, that PD-L1 blockade re-sensitized PARPi-treated cancer cells to T-cell killing, and the combination of PARPi and anti-PD-L1 therapy demonstrated greater antitumor activity and tumor control compared with each agent alone (127), further indicating a potential synergistic effect of combination DNA damage response inhibitors (DDRi's) and ICI. Combination PARPi with PD-1/PDL1 targeted therapies demonstrated increased TILs and enhanced antitumor immunity in both BRCA-proficient and BRCA-deficient mouse models of TNBC $(123,127)$, indicating additional PARPimediated immunologic factors associated with ICI outcomes. Interestingly, whole exome sequencing of cancer patients previously treated with $\mathrm{PD}-1$ inhibitors revealed that ICI responders are enriched for BRCA mutations (8). Altogether, this dual effect of DDRi-induced immune activation and PD-L1dependent immunosuppression suggests immunologic vulnerability that may be exploited through the use of ICI, and serves as the rationale for studies investigating the clinical efficacy of combination therapy with PARPi's and anti-PD-1/PD-L1 in multiple cancers, including TNBC $(121,128)$.

A summary of ongoing clinical trials combining DDR targeting agents with immunotherapy is listed in Table $\mathbf{1}$.

\subsection{Exploiting BRCA1/2 Deficiency and Immunotherapy in TNBC}

Given the potential of tumor cell HR defects, including BRCAness, to increase susceptibility to ICI through enhanced immune activation and expression of PD-1 or PD-L1 (103), ICI response is being studied in cancers, including breast cancers, with germline mutations in BRCA1 or BRCA2 (NCT01772004, NCT03025035). In previously treated, platinum-resistant recurrent ovarian cancer, Durvalumab and Olaparib demonstrated clinical activity, irrespective of BRCA mutation status (NCT02484404) (129). Interestingly, analysis of core biopsy and blood samples revealed 
TABLE 1 | Ongoing clinical trials of combination DNA targeting and/or immunotherapy agents in TNBC or BC with DDR mutations.

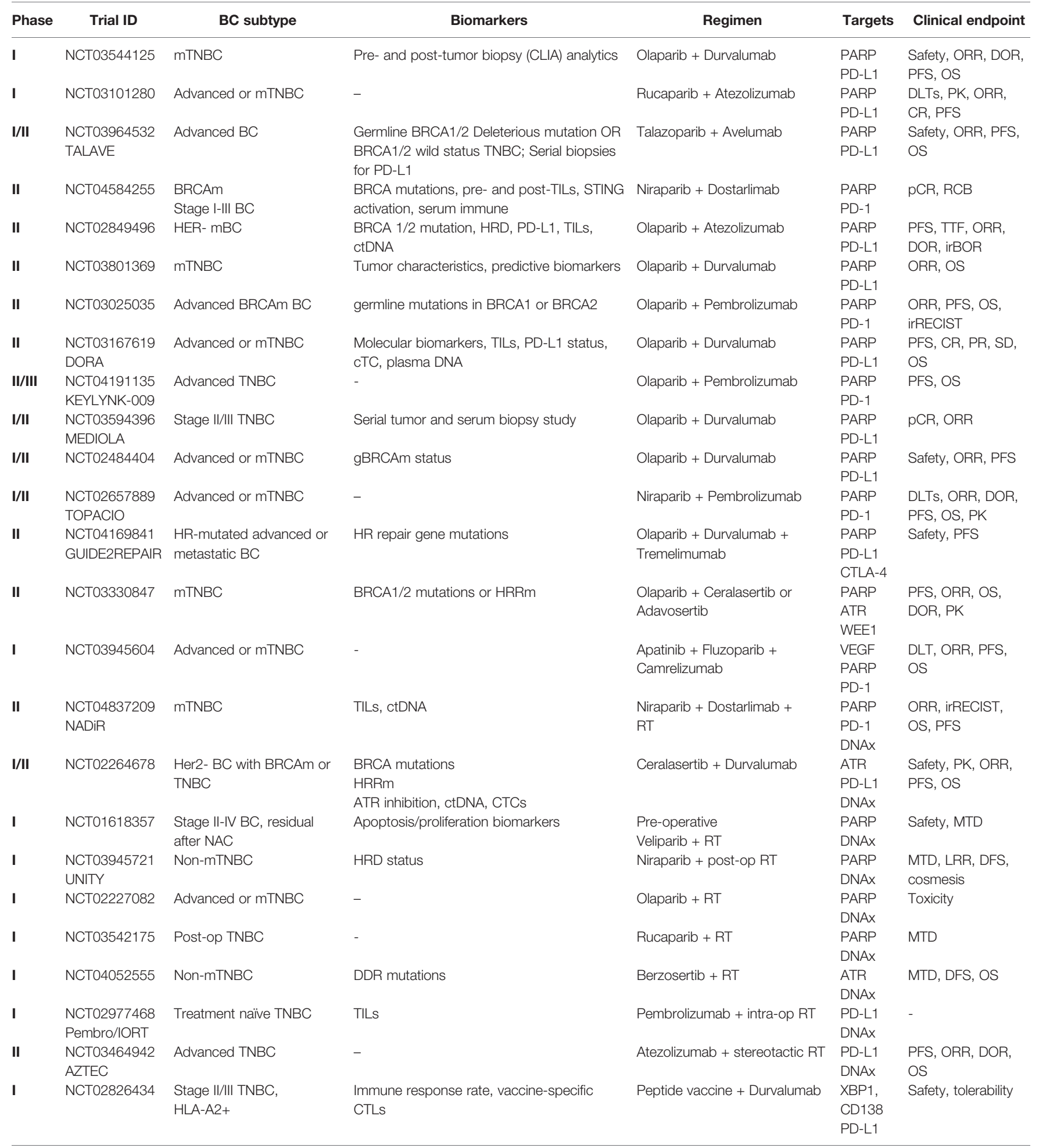

mTNBC, Metastatic triple-negative breast cancer; BC, breast cancer; (CLIA) analytics, Proportion of completion of Clinical Laboratory Improvement Act; ORR, objective response rate; DOR, duration of response; PFS, progression-free survival; OS, overall survival; DLTS, Dose-Limiting Toxicities; MTD, maximum tolerated dose; PK, Pharmacokinetics; pCR, pathologic complete response; TILS, tumor infiltrating lymphocytes; STING, stimulator of interferon genes; RCB, pathway, residual cancer burden; HRD, homologous recombination deficiencies; HRRm, HRR-related gene mutation; CtDNA, circulating tumor DNA; TTF, time to treatment failure; irBOR, immune-related best overall response; irRECIST, immune-related; cTC, circulating tumor cells; SD, stable disease; gBRCAm, germline BRCA1 and BRCA2 mutation; DDR, DNA-damage response; DCR, pathway, disease control rate; LRR, locoregional relapse; DFS, distant relapse; CTLs, Cytotoxic T Lymphocytes; DNAx, therapeutic targeting of DNA DSBs; (HLA)-A2+, Human Leukocyte Antigen. 
this combination created a stronger immunostimulatory phenotype with enhanced IFN $-\gamma$ and CXCL9/CXCL10 expression, systemic IFN- $\gamma /$ TNF- $\alpha$ production and TILs (128, 129). Combination treatment with Durvalumab with the PARPi Olaparib is currently under exploration in a phase I/II trial of women's cancers, including patients with TNBC, with biomarker evaluation ongoing (128). The phase II MEDIOLA basket trial assessed the efficacy and safety of combination Olaparib and Durvalumab in patients with solid tumors, including ovarian cancer, breast cancer and gastric cancer (NCT02734004). In germline BRCA mutant, platinum-sensitive relapsed ovarian cancer, this combination demonstrated an overall response rate (ORR) of $63 \%$ and a 12 -week disease control rate (DCR) of $81 \%$ (15). In gBRCAm HER2 negative metastatic breast cancer, the DCR was $80 \%$ at 12 weeks and $50 \%$ at 28 weeks, with ORR of $63 \%$. Median PFS (mPFS) was 9.2 months and median overall survival (mOS) was 21.5 months. Moreover, patients with no prior line of chemotherapy had higher ORR and longer OS than those with two prior lines (respectively $78 \%$ vs. 50\% for ORR and 21.3 vs. 16.9 months for OS) (15). Although there is no observed association between PD-L1 positivity and TILs at this point in the trial, there was a trend of higher PD-L1 and increased TILs observed in archival samples in patients who had SD/PR/CR, which was not observed in patients with progressive disease. Furthermore, high PD-L1 was observed in patients with DCR at 12 weeks $(15,33)$. In the phase II TOPACIO trial (NCT02657889), Niraparib and Pembrolizumab combination therapy has demonstrated clinical benefit in platinum-resistant TNBC, with numerically higher response rates in those with BRCA-mutated TNBC tumors (ORR of BRCAm vs. BRCA wild-type, 47\% vs. 11\%) (130). A phase II multicenter study of Durvalumab and Olaparib is underway for patients with advanced TNBC that is inoperable, locally advanced, or metastatic, and is not amenable to resection with curative intent, and who have received at least 4 cycles of platinum-based chemotherapy with demonstrated clinical benefit (NCT03167619). Other trials combining PARPi's, including Olaparib, Rucaparib, and Fluzoparib, with ICIs, such as Pembrolizumab (NCT03101280), Atezolizumab (NCT04191135), and Camrelizumab (NCT03945604), respectively, for locally advanced or metastatic TNBC are also underway. A phase II study will evaluate safety and efficacy of combination of PARPi (niraparib), PD-1 mAb (Dostarlimab), and RT in metastatic TNBC (NCT04837209). Though the relationship between endogenous or PARPi-induced BRCAness and immunotherapy response is still being investigated, these ongoing clinical trials will help establish the effect HR-deficiency and DDR targeting therapies on ICI outcomes in TNBC.

\subsection{Other DDR Targets and Immunotherapy in TNBC}

Evidence that unrepaired DNA damage induced by PARPi expands the anti-tumor activity of the ICI has prompted investigation of other key mediators implied in DNA replication and repair, such as ATM, ATR, CHK1, CHK2, DNA-PK, and WEE1 (31, 120, 121). Given the immunomodulatory effects seen with PARPi, these additional DDR mediators are exciting targets for combined immunotherapy. In preclinical breast cancer studies, the combination of a selective ATR inhibitor with Avelumab and platinum-based chemotherapy resulted in antitumor effect in syngeneic tumor models, leading to overall survival benefit compared to any dual-combination group, and also provided protective antitumor immunity with immunological memory in cured mice (131). In a preclinical model of lung cancer, CHK1 inhibition potentiated the antitumor effect of PD-L1 blockade and augmented cytotoxic T cell infiltration (27). In other studies, inhibition of DNA-PK upregulated PD-L1 in a cGAS-STING-dependent manner in irradiated p53-mutant cancer cells, suggesting selective blockade of NHEJ repair of DSB exhibits immunomodulatory effects similar to those seen in HR-inhibition. Preclinical studies of combined DNA-PK inhibition, radiation and PD-L1 blockade demonstrated increased anti-tumor activity in a p53-mutant cancer, suggesting that inhibition of DNA-PK inhibits repair of radiation-induced DSBs resulting in potentiation of anti-tumor immunity, adaptive PD-L1 expression through DDR-dependent mechanisms, and subsequent responsiveness to immune checkpoint blockade (132).

These promising preclinical studies have led to several early phase clinical trials. A clinical study in patients with advanced or metastatic cancers, use of Ceralasertib, a potent and selective ATR inhibitor in combination with Durvalumab is being evaluated (NCT02264678). A selective ATR kinase inhibitor, AZD6738, is undergoing a phase II study with Olaparib for metastatic TNBC patients with BRCA1/2 mutations or HRD (NCT03330847). The phase Ib BISCAY study, Durvalumab and Olaparib or the WEE1 inhibitor Adavosertib in patients with metastatic cancer with any detected HR-deficiency (NCT02546661). A phase I study combining the CHK1 inhibitor, Prexasertib, with a PD-L1 mAb demonstrated the potential for enhanced therapeutic activity and increased cytotoxic T cell activation (125).

Further highlighting the indication that DDR-inhibitors and DNA-damaging agents may enhance immunotherapeutic response, a phase II clinical trial is evaluating the efficacy of Atezolizumab with stereotactic RT for advanced TNBC (NCT03464942), and a phase I study for the feasibility of adjuvant Durvalumab with a peptide vaccine is underway for patients with stage II and III TNBC after completion of standard adjuvant therapy (NCT02826434). Altogether, early studies indicate a potential therapeutic benefit of DDR-pathway targeting/inhibition in combination with immunotherapy, and ongoing trials will provide new insights into and establish clinical efficacy of the immune potentiating efficacy of DDR-inhibitors.

\section{SUMMARY}

TNBC represents a highly diverse set of breast cancers with complicated molecular and immunologic landscapes, and thus remains a challenging oncologic entity to tackle effectively. However, advances in genomic profiling and our understanding of the interplay between DNA damage response and cancer immunity has resulted in exciting immuno-molecular 
therapeutic opportunities. Of these, DDR-deficiencies including BRCAness have been shown to promote immunologic vulnerability through DNA damage-induced high TMB, immune-stimulatory and suppressive features, as well as adaptive immune resistance via $\mathrm{PD}-\mathrm{L} 1$ upregulation. DDR deficiencies represent a frequent aberration in TNBC, and exploitation of immunologic consequences offers potential therapeutic leverage that combines favorable immune effects of DNA/DDR-targeted therapies with restoration of cytotoxic antitumor immune cells. The role for endogenous as well as therapyinduced DNA damage signaling in PD-L1 induced expression, and the possibility of circumventing DNA targeted therapy-induced immune suppression with concomitant immunotherapy provide rationale for combining agents targeting the DDR and the immune system. Immunotherapy, chiefly ICI, represents an opportunity to flip the switch back to immune activation, particularly in the context of concomitant DDR pathway targeting therapies, such as PARP inhibitors and others.

PARP inhibitor monotherapies, as well as therapeutic combinations, have demonstrated promising clinical benefit, and their effects on enhancing lethal DNA damage vulnerabilities have been shown. Nonetheless, the underlying mechanisms of PARPimediated sensitization of tumors to immunotherapy and/or radiotherapy remain to be fully elucidated. Furthermore, rapid translation of these potential breakthroughs in TNBC treatment will require thoughtful incorporation and thorough dissection of clinical trial outcomes and their implications into everyday clinical practice. Despite preclinical and clinical studies that have demonstrated PARPi-mediated immunosuppression via $\mathrm{PD}-\mathrm{L} 1$ induction and complementary restoration of PARPi sensitivity via PD-L1 inhibition, with the added possibility of enhanced antitumor immunity, many unanswered questions remain regarding the potential benefit of combined targeted therapies and ICIs in TNBC. In addition to PARPi, other repair pathway mediators such as ATR, and CHK1, are being investigated in combination with immune-based strategies, and thus careful consideration of promising therapeutics as well as other immunotherapeutic strategies in the pipeline should not be overlooked. Optimization of treatment schemas for combined immunotherapeutic strategies remains a challenge, as does validation of biomarkers that will identify which patients will most benefit from either PARP

\section{REFERENCES}

1. Garrido-Castro AC, Lin NU, Polyak K. Insights Into Molecular Classifications of Triple-Negative Breast Cancer: Improving Patient Selection for Treatment. Cancer Discov (2019) 9:176-98. doi: 10.1158/ 2159-8290.CD-18-1177

2. Bianchini G, Balko JM, Mayer IA, Sanders ME, Gianni L. Triple-Negative Breast Cancer: Challenges and Opportunities of a Heterogeneous Disease. Nat Rev Clin Oncol (2016) 13:674-90. doi: 10.1038/nrclinonc.2016.66

3. Schmid P, Adams S, Rugo HS, Schneeweiss A, Barrios CH, Iwata $\mathrm{H}$, et al. Atezolizumab and Nab-Paclitaxel in Advanced Triple-Negative Breast Cancer. N Engl J Med (2018) 379:2108-21. doi: 10.1056/NEJMoa1809615

4. Jiang YZ, Ma D, Suo C, Shi J, Xue M, Hu X, et al. Genomic and Transcriptomic Landscape of Triple-Negative Breast Cancers: Subtypes and Treatment Strategies. Cancer Cell (2019) 35:428-40.e425. doi: 10.1016/j.ccell.2019.02.001 inhibitors in combination with immunotherapy, radiotherapy, or other targeted therapies.

Lastly, identifying additional key mediators of DNA damageassociated immune modulation that regulate disease progression, therapeutic response and resistance will require further preclinical investigation and careful analysis of clinical samples to assess DDR deficiencies in certain tumor subsets, with the ultimate goal of personalizing DNA targeting and immune-based therapies in combination with conventional DNA- and immuneaugmenting therapies, such as chemotherapy and radiation, to maximize the combined benefits of each approach and effectively target immunosuppressive pathways that contribute to immune escape and tumor progression. It will also be important to identify mediators of poor response to ICIs and improved prognostic markers for existing therapies to select patients that may benefit from alternative therapeutic strategies and explore options for TNBC refractory to ICI or PD-L1 negative TNBC. Furthermore, the role of less studied DDR mechanisms related to ICI is still unclear, and future work is needed to better predict which DNA damage response and repair pathways are most suitable for therapeutic targeting in specific subsets of patients.

\section{AUTHOR CONTRIBUTIONS}

Both authors initiated the concept and wrote the manuscript. All authors contributed to the article and approved the submitted version.

\section{FUNDING}

This work was supported by the UAB Department of Radiation Oncology.

\section{ACKNOWLEDGMENTS}

The authors would like to thank Anne P. Branum for her assistance in figure graphic design.

5. Bai X, Ni J, Beretov J, Graham P, Li Y. Triple-Negative Breast Cancer Therapeutic Resistance: Where Is the Achilles' Heel? Cancer Lett (2021) 497:100-11. doi: 10.1016/j.canlet.2020.10.016

6. Hou Y, Nitta H, Parwani AV, Li Z. PD-L1 and CD8 Are Associated With Deficient Mismatch Repair Status in Triple-Negative and HER2-Positive Breast Cancers. Hum Pathol (2019) 86:108-14. doi: 10.1016/j.humpath.2018.12.007

7. Lee KJ, Piett CG, Andrews JF, Mann E, Nagel ZD, Gassman NR. Defective Base Excision Repair in the Response to DNA Damaging Agents in Triple Negative Breast Cancer. PloS One (2019) 14:e0223725. doi: 10.1371/ journal.pone.0223725

8. Staaf J, Glodzik D, Bosch A, Vallon-Christersson J, Reutersward C, Hakkinen J, et al. Whole-Genome Sequencing of Triple-Negative Breast Cancers in a Population-Based Clinical Study. Nat Med (2019) 25:1526-33. doi: 10.1038/s41591-019-0582-4

9. Patel SA, Meyer JR, Greco SJ, Corcoran KE, Bryan M, Rameshwar P. Mesenchymal Stem Cells Protect Breast Cancer Cells Through Regulatory T 
Cells: Role of Mesenchymal Stem Cell-Derived TGF-Beta. J Immunol (2010) 184:5885-94. doi: 10.4049/jimmunol.0903143

10. Sami E, Paul BT, Koziol JA, ElShamy WM. The Immunosuppressive Microenvironment in BRCA1-IRIS-Overexpressing TNBC Tumors Is Induced by Bidirectional Interaction With Tumor-Associated Macrophages. Cancer Res (2020) 80:1102-17. doi: 10.1158/00085472.CAN-19-2374

11. Black M, Barsoum IB, Truesdell P, Cotechini T, Macdonald-Goodfellow SK, Petroff M, et al. Activation of the PD-1/PD-L1 Immune Checkpoint Confers Tumor Cell Chemoresistance Associated With Increased Metastasis. Oncotarget (2016) 7:10557-67. doi: 10.18632/oncotarget.7235

12. Mori H, Kubo M, Yamaguchi R, Nishimura R, Osako T, Arima N, et al. The Combination of PD-L1 Expression and Decreased Tumor-Infiltrating Lymphocytes Is Associated With a Poor Prognosis in Triple-Negative Breast Cancer. Oncotarget (2017) 8:15584-92. doi: 10.18632/oncotarget.14698

13. Wen WX, Leong C-O. Association of BRCA1- and BRCA2-Deficiency With Mutation Burden, Expression of PD-L1/PD-1, Immune Infiltrates, and T Cell-Inflamed Signature in Breast Cancer. PloS One (2019) 14:e0215381. doi: 10.1371/journal.pone.0215381

14. Litton JK, Rugo HS, Ettl J, Hurvitz SA, Goncalves A, Lee KH, et al. Talazoparib in Patients With Advanced Breast Cancer and a Germline BRCA Mutation. N Engl J Med (2018) 379:753-63. doi: 10.1056/NEJMoa1802905

15. Domchek S, Postel-Vinay S, Im SA, Park YH, Delord JP, Italiano A, et al. 1191ophase II Study of Olaparib (O) and Durvalumab (D) (MEDIOLA): Updated Results in Patients (Pts) With Germline BRCA-Mutated (gBRCAm) Metastatic Breast Cancer (MBC). Ann Oncol (2019) 30:v477. doi: 10.1093/annonc/mdz253.017

16. Robson M, Im SA, Senkus E, Xu B, Domchek SM, Masuda N, et al. Olaparib for Metastatic Breast Cancer in Patients With a Germline BRCA Mutation. N Engl J Med (2017) 377:523-33. doi: 10.1056/NEJMoa1706450

17. Tung NM, Robson ME, Ventz S, Santa-Maria CA, Nanda R, Marcom PK, et al. TBCRC 048: Phase II Study of Olaparib for Metastatic Breast Cancer and Mutations in Homologous Recombination-Related Genes. J Clin Oncol (2020) 38:4274-82. doi: 10.1200/JCO.20.02151

18. Yarchoan M, Hopkins A, Jaffee EM. Tumor Mutational Burden and Response Rate to PD-1 Inhibition. N Engl J Med (2017) 377:2500-1. doi: 10.1056/NEJMc1713444

19. Loi S, Drubay D, Adams S, Pruneri G, Francis PA, Lacroix-Triki M, et al. Tumor-Infiltrating Lymphocytes and Prognosis: A Pooled Individual Patient Analysis of Early-Stage Triple-Negative Breast Cancers. J Clin Oncol (2019) 37:559-69. doi: 10.1200/JCO.18.01010

20. Telli ML, Chu C, Badve SS, Vinayak S, Silver DP, Isakoff SJ, et al. Association of Tumor-Infiltrating Lymphocytes With Homologous Recombination Deficiency and BRCA1/2 Status in Patients With Early Triple-Negative Breast Cancer: A Pooled Analysis. Clin Cancer Res (2020) 26:2704-10. doi: 10.1158/1078-0432.CCR-19-0664

21. Pellegrino B, Musolino A, Llop-Guevara A, Serra V, De Silva P, Hlavata Z, et al. Homologous Recombination Repair Deficiency and the Immune Response in Breast Cancer: A Literature Review. Transl Oncol (2020) 13:410-22. doi: 10.1016/j.tranon.2019.10.010

22. Barroso-Sousa R, Keenan TE, Pernas S, Exman P, Jain E, Garrido-Castro AC, et al. Tumor Mutational Burden and PTEN Alterations as Molecular Correlates of Response to PD-1/L1 Blockade in Metastatic Triple-Negative Breast Cancer. Clin Cancer Res (2020) 26:2565-72. doi: 10.1158/10780432.CCR-19-3507

23. Savas $P$, Loi S. Expanding the Role for Immunotherapy in Triple-Negative Breast Cancer. Cancer Cell (2020) 37:623-4. doi: 10.1016/j.ccell.2020.04.007

24. Green AR, Aleskandarany MA, Ali R, Hodgson EG, Atabani S, De Souza K, et al. Clinical Impact of Tumor DNA Repair Expression and T-Cell Infiltration in Breast Cancers. Cancer Immunol Res (2017) 5:292-9. doi: 10.1158/2326-6066.CIR-16-0195

25. Lamberti G, Andrini E, Sisi M, Federico AD, Ricciuti B. Targeting DNA Damage Response and Repair Genes to Enhance Anticancer Immunotherapy: Rationale and Clinical Implication. Future Oncol (2020) 16:1751-66. doi: 10.2217/fon-2020-0215

26. Sato H, Niimi A, Yasuhara T, Permata TBM, Hagiwara Y, Isono M, et al. DNA Double-Strand Break Repair Pathway Regulates PD-L1 Expression in Cancer Cells. Nat Commun (2017) 8:1751. doi: 10.1038/s41467-017-01883-9
27. Sen T, Rodriguez BL, Chen L, Corte CD, Morikawa N, Fujimoto J, et al. Targeting DNA Damage Response Promotes Anti-Tumor Immunity Through STING-Mediated T-Cell Activation in Small Cell Lung Cancer. Cancer Discov (2019) 9:CD-18-1020. doi: 10.1158/2159-8290.cd-18-1020

28. Lord CJ, Ashworth A. The DNA Damage Response and Cancer Therapy. Nature (2012) 481:287-94. doi: 10.1038/nature10760

29. Burrell RA, McGranahan N, Bartek J, Swanton C. The Causes and Consequences of Genetic Heterogeneity in Cancer Evolution. Nature (2013) 501:338-45. doi: 10.1038/nature12625

30. Blackford AN, Jackson SP. ATM, ATR, and DNA-PK: The Trinity at the Heart of the DNA Damage Response. Mol Cell (2017) 66:801-17. doi: 10.1016/j.molcel.2017.05.015

31. Do K, Doroshow JH, Kummar S. Weel Kinase as a Target for Cancer Therapy. Cell Cycle (2013) 12:3348-53. doi: 10.4161/cc.26062

32. Tubbs A, Nussenzweig A. Endogenous DNA Damage as a Source of Genomic Instability in Cancer. Cell (2017) 168:644-56. doi: 10.1016/j.cell.2017.01.002

33. Sayaman RW, Saad M, Thorsson V, Hu D, Hendrickx W, Roelands J, et al. Germline Genetic Contribution to the Immune Landscape of Cancer. Immunity (2021) 54:367-86.e368. doi: 10.1016/j.immuni.2021.01.011

34. Lord CJ, Ashworth A. PARP Inhibitors: Synthetic Lethality in the Clinic. Science (2017) 355:1152-8. doi: 10.1126/science.aam7344

35. Farmer H, McCabe N, Lord CJ, Tutt AN, Johnson DA, Richardson TB, et al. Targeting the DNA Repair Defect in BRCA Mutant Cells as a Therapeutic Strategy. Nature (2005) 434:917-21. doi: 10.1038/nature03445

36. Bryant HE, Schultz N, Thomas HD, Parker KM, Flower D, Lopez E, et al. Specific Killing of BRCA2-Deficient Tumours With Inhibitors of Poly(ADPRibose) Polymerase. Nature (2005) 434:913-7. doi: 10.1038/nature03443

37. Golan T, Hammel P, Reni M, Cutsem EV, Macarulla T, Hall MJ, et al. Maintenance Olaparib for Germline BRCA-Mutated Metastatic Pancreatic Cancer. New Engl J Med (2019) 381:317-27. doi: 10.1056/NEJMoa1903387

38. Min A, Im S-A. PARP Inhibitors as Therapeutics: Beyond Modulation of PARylation. Cancers (2020) 12:394. doi: 10.3390/cancers12020394

39. Litton JK, Hurvitz SA, Mina LA, Rugo HS, Lee KH, Goncalves A, et al. Talazoparib Versus Chemotherapy in Patients With Germline BRCA1/2Mutated HER2-Negative Advanced Breast Cancer: Final Overall Survival Results From the EMBRACA Trial. Ann Oncol (2020) 31:1526-35. doi: 10.1016/j.annonc.2020.08.2098

40. Murai J, S-yN H, BB D, Renaud A, Zhang Y, JH D, et al. Trapping of PARP1 and PARP2 by Clinical PARP Inhibitors. Cancer Res (2012) 72:5588-99. doi: 10.1158/0008-5472.CAN-12-2753

41. Shen J, Zhao W, Ju Z, Wang L, Peng Y, Labrie M, et al. PARPi Triggers the STING-Dependent Immune Response and Enhances the Therapeutic Efficacy of Immune Checkpoint Blockade Independent of BRCAness. Cancer Res (2019) 79:311-9. doi: 10.1158/0008-5472.CAN-18-1003

42. Loibl S, Weber KE, Timms KM, Elkin EP, Hahnen E, Fasching PA, et al. Survival Analysis of Carboplatin Added to an Anthracycline/Taxane-Based Neoadjuvant Chemotherapy and HRD Score as Predictor of Response-Final Results From GeparSixto. Ann Oncol (2018) 29:2341-7. doi: 10.1093/ annonc/mdy460

43. Tu X, Kahila MM, Zhou Q, Yu J, Kalari KR, Wang L, et al. ATR Inhibition Is a Promising Radiosensitizing Strategy for Triple-Negative Breast Cancer. Mol Cancer Ther (2018) 17:2462-72. doi: 10.1158/1535-7163.MCT-18-0470

44. Alotaibi M, Sharma K, Saleh T, Povirk LF, Hendrickson EA, Gewirtz DA. Radiosensitization by PARP Inhibition in DNA Repair Proficient and Deficient Tumor Cells: Proliferative Recovery in Senescent Cells. Radiat Res (2016) 185:229-45. doi: 10.1667/RR14202.1

45. Isakoff SJ, Puhalla S, Domchek SM, Friedlander M, Kaufman B, Robson M, et al. A Randomized Phase II Study of Veliparib With Temozolomide or Carboplatin/Paclitaxel Versus Placebo With Carboplatin/Paclitaxel in BRCA1/2 Metastatic Breast Cancer: Design and Rationale. Future Oncol (2017) 13:307-20. doi: 10.2217/fon-2016-0412

46. Somlo G, Frankel PH, Arun BK, Ma CX, Garcia AA, Cigler T, et al. Efficacy of the PARP Inhibitor Veliparib With Carboplatin or as a Single Agent in Patients With Germline BRCA1- or BRCA2-Associated Metastatic Breast Cancer: California Cancer Consortium Trial Nct01149083. Clin Cancer Res (2017) 23:4066-76. doi: 10.1158/1078-0432.CCR-16-2714

47. Kummar S, Wade JL, Oza AM, Sullivan D, Chen AP, Gandara DR, et al. Randomized Phase II Trial of Cyclophosphamide and the Oral Poly (ADP- 
Ribose) Polymerase Inhibitor Veliparib in Patients With Recurrent, Advanced Triple-Negative Breast Cancer. Invest New Drugs (2016) 34:355-63. doi: 10.1007/s10637-016-0335-x

48. Wang H, Yee D. I-SPY 2: A Neoadjuvant Adaptive Clinical Trial Designed to Improve Outcomes in High-Risk Breast Cancer. Curr Breast Cancer Rep (2019) 11:303-10. doi: 10.1007/s12609-019-00334-2

49. Severson TM, Wolf DM, Yau C, Peeters J, Wehkam D, Schouten PC, et al. The BRCA1ness Signature is Associated Significantly With Response to PARP Inhibitor Treatment Versus Control in the I-SPY 2 Randomized Neoadjuvant Setting. Breast Cancer Research: BCR (2017) 19:99. doi: 10.1186/s13058-017-0861-2

50. Sikov WM, Berry DA, Perou CM, Singh B, Cirrincione CT, Tolaney SM, et al. Impact of the Addition of Carboplatin and/or Bevacizumab to Neoadjuvant Once-Per-Week Paclitaxel Followed by Dose-Dense Doxorubicin and Cyclophosphamide on Pathologic Complete Response Rates in Stage II to III Triple-Negative Breast Cancer: CALGB 40603 (Alliance). J Clin Oncol (2015) 33:13-21. doi: 10.1200/JCO.2014.57.0572

51. von Minckwitz G, Schneeweiss A, Loibl S, Salat C, Denkert C, Rezai M, et al. Neoadjuvant Carboplatin in Patients With Triple-Negative and HER2Positive Early Breast Cancer (GeparSixto; GBG 66): A Randomised Phase 2 Trial. Lancet Oncol (2014) 15:747-56. doi: 10.1016/S1470-2045(14)70160-3

52. Nowsheen S, Cooper T, Stanley JA, Yang ES. Synthetic Lethal Interactions Between EGFR and PARP Inhibition in Human Triple Negative Breast Cancer Cells. PloS One (2012) 7:e46614. doi: 10.1371/journal.pone.0046614

53. Verma N, Vinik Y, Saroha A, Nair NU, Ruppin E, Mills G, et al. Synthetic Lethal Combination Targeting BET Uncovered Intrinsic Susceptibility of TNBC to Ferroptosis. Sci Adv (2020) 6. doi: 10.1126/sciadv.aba8968

54. Li Y, Wang Y, Zhang W, Wang X, Chen L, Wang S. BKM120 Sensitizes BRCA-Proficient Triple Negative Breast Cancer Cells to Olaparib Through Regulating FOXM1 and Exo1 Expression. Sci Rep (2021) 11:4774. doi: 10.1038/s41598-021-82990-y

55. Stringer-Reasor EM, May JE, Olariu E, Caterinicchia V, Li Y, Chen D, et al. An Open-Label, Pilot Study of Veliparib and Lapatinib in Patients With Metastatic, Triple-Negative Breast Cancer. Breast Cancer Research: BCR (2021) 23:30. doi: 10.1186/s13058-021-01408-9

56. Prasad V, Kaestner V, Mailankody S. Cancer Drugs Approved Based on Biomarkers and Not Tumor Type-FDA Approval of Pembrolizumab for Mismatch Repair-Deficient Solid Cancers. JAMA Oncol (2018) 4:157-8. doi: 10.1001/jamaoncol.2017.4182

57. Mills AM, Dill EA, Moskaluk CA, Dziegielewski J, Bullock TN, Dillon PM. The Relationship Between Mismatch Repair Deficiency and PD-L1 Expression in Breast Carcinoma. Am J Surg Pathol (2018) 42:183-91. doi: 10.1097/PAS.0000000000000949

58. Liang Y, Li Y, Song X, Zhang N, Sang Y, Zhang H, et al. Long Noncoding RNA LINP1 Acts as an Oncogene and Promotes Chemoresistance in Breast Cancer. Cancer Biol Ther (2018) 19:120-31. doi: 10.1080/15384047.2017.1394543

59. Zhang $\mathrm{Y}, \mathrm{He} \mathrm{Q}, \mathrm{Hu} Z$, Feng $\mathrm{Y}$, Fan L, Tang Z, et al. Long Noncoding RNA LINP1 Regulates Repair of DNA Double-Strand Breaks in Triple-Negative Breast Cancer. Nat Struct Mol Biol (2016) 23:522-30. doi: 10.1038/ nsmb.3211

60. Lamb R, Fiorillo M, Chadwick A, Ozsvari B, Reeves KJ, Smith DL, et al. Doxycycline Down-Regulates DNA-PK and Radiosensitizes Tumor Initiating Cells: Implications for More Effective Radiation Therapy. Oncotarget (2015) 6:14005-25. doi: 10.18632/oncotarget.4159

61. Hu ZI, Ho AY, McArthur HL. Combined Radiation Therapy and Immune Checkpoint Blockade Therapy for Breast Cancer. Int J Radiat Oncol Biol Phys (2017) 99:153-64. doi: 10.1016/j.ijrobp.2017.05.029

62. Citrin DE. Recent Developments in Radiotherapy. N Engl J Med (2017) 377:2200-1. doi: 10.1056/NEJMra1608986

63. Matsumura S, Wang B, Kawashima N, Braunstein S, Badura M, Cameron TO, et al. Radiation-Induced CXCL16 Release by Breast Cancer Cells Attracts Effector T Cells. J Immunol (2008) 181:3099-107. doi: 10.4049/ jimmunol.181.5.3099

64. Reislander T, Groelly FJ, Tarsounas M. DNA Damage and Cancer Immunotherapy: A STING in the Tale. Mol Cell (2020) 80:21-8. doi: 10.1016/j.molcel.2020.07.026

65. Deng L, Liang H, Xu M, Yang X, Burnette B, Arina A, et al. STINGDependent Cytosolic DNA Sensing Promotes Radiation-Induced Type I
Interferon-Dependent Antitumor Immunity in Immunogenic Tumors. Immunity (2014) 41:843-52. doi: 10.1016/j.immuni.2014.10.019

66. Dutt S, Ahmed MM, Loo BW Jr, Strober S. Novel Radiation Therapy Paradigms and Immunomodulation: Heresies and Hope. Semin Radiat Oncol (2020) 30:194-200. doi: 10.1016/j.semradonc.2019.12.006

67. Burnette BC, Liang H, Lee Y, Chlewicki L, Khodarev NN, Weichselbaum RR, et al. The Efficacy of Radiotherapy Relies Upon Induction of Type I Interferon-Dependent Innate and Adaptive Immunity. Cancer Res (2011) 71:2488-96. doi: 10.1158/0008-5472.CAN-10-2820

68. Shevtsov M, Sato H, Multhoff G, Shibata A. Novel Approaches to Improve the Efficacy of Immuno-Radiotherapy. Front Oncol (2019) 9:156. doi: $10.3389 /$ fonc. 2019.00156

69. Parkes EE, Walker SM, Taggart LE, McCabe N, Knight LA, Wilkinson R, et al. Activation of STING-Dependent Innate Immune Signaling By S-PhaseSpecific DNA Damage in Breast Cancer. J Natl Cancer Inst (2017) 109. doi 10.1093/jnci/djw199

70. Benci JL, Xu B, Qiu Y, Wu TJ, Dada H, Twyman-Saint Victor C, et al. Tumor Interferon Signaling Regulates a Multigenic Resistance Program to Immune Checkpoint Blockade. Cell (2016) 167:1540-54.e1512. doi: 10.1016/ j.cell.2016.11.022

71. Vendetti FP, Karukonda P, Clump DA, Teo T, Lalonde R, Nugent K, et al. ATR Kinase Inhibitor AZD6738 Potentiates CD8+ T Cell-Dependent Antitumor Activity Following Radiation. J Clin Invest (2018) 128:3926-40. doi: 10.1172/JCI96519

72. Ho AY, Barker CA, Arnold BB, Powell SN, Hu ZI, Gucalp A, et al. A Phase 2 Clinical Trialassessing Theefficacy and Safety of Pembrolizumab and Radiotherapy in Patients With Metastatic Triple-Negative Breast Cancer. Cancer (2020) 126:850-60. doi: 10.1002/cncr.32599

73. Loap P, Loirat D, Berger F, Ricci F, Vincent-Salomon A, Ezzili C, et al. Combination of Olaparib and Radiation Therapy for Triple Negative Breast Cancer: Preliminary Results of the RADIOPARP Phase 1 Trial. Int J Radiat Oncol Biol Phys (2021) 109:436-40. doi: 10.1016/j.ijrobp.2020.09.032

74. Leon-Ferre RA, Polley MY, Liu H, Gilbert JA, Cafourek V, Hillman DW, et al. Impact of Histopathology, Tumor-Infiltrating Lymphocytes, and Adjuvant Chemotherapy on Prognosis of Triple-Negative Breast Cancer. Breast Cancer Res Treat (2018) 167:89-99. doi: 10.1007/s10549-017-4499-7

75. Molinero L, Li Y, Chang CW, Maund S, Berg M, Harrison J, et al. Tumor Immune Microenvironment and Genomic Evolution in a Patient With Metastatic Triple Negative Breast Cancer and a Complete Response to Atezolizumab. I Immunother Cancer (2019) 7:274. doi: 10.1186/s40425019-0740-8

76. Karn T, Jiang T, Hatzis C, Sanger N, El-Balat A, Rody A, et al. Association Between Genomic Metrics and Immune Infiltration in Triple-Negative Breast Cancer. JAMA Oncol (2017) 3:1707-11. doi: 10.1001/jamaoncol. 2017.2140

77. Adams S, Diamond JR, Hamilton E, Pohlmann PR, Tolaney SM, Chang CW, et al. Atezolizumab Plus Nab-Paclitaxel in the Treatment of Metastatic Triple-Negative Breast Cancer With 2-Year Survival Follow-Up: A Phase 1b Clinical Trial. JAMA Oncol (2019) 5:334-42. doi: 10.1001/jamaoncol. 2018.5152

78. Tomioka N, Azuma M, Ikarashi M, Yamamoto M, Sato M, Watanabe KI, et al. The Therapeutic Candidate for Immune Checkpoint Inhibitors Elucidated by the Status of Tumor-Infiltrating Lymphocytes (TILs) and Programmed Death Ligand 1 (PD-L1) Expression in Triple Negative Breast Cancer (TNBC). Breast Cancer (2018) 25:34-42. doi: 10.1007/s12282-0170781-0

79. Denkert C, von Minckwitz G, Brase JC, Sinn BV, Gade S, Kronenwett R, et al. Tumor-Infiltrating Lymphocytes and Response to Neoadjuvant Chemotherapy With or Without Carboplatin in Human Epidermal Growth Factor Receptor 2-Positive and Triple-Negative Primary Breast Cancers. J Clin Oncol (2015) 33:983-91. doi: 10.1200/JCO.2014.58.1967

80. Adams S, Gray RJ, Demaria S, Goldstein L, Perez EA, Shulman LN, et al. Prognostic Value of Tumor-Infiltrating Lymphocytes in Triple-Negative Breast Cancers From Two Phase III Randomized Adjuvant Breast Cancer Trials: ECOG 2197 and ECOG 1199. J Clin Oncol (2014) 32:2959-66. doi 10.1200/JCO.2013.55.0491

81. Liu F, Lang R, Zhao J, Zhang X, Pringle GA, Fan Y, et al. CD8(+) Cytotoxic T Cell and FOXP3(+) Regulatory $\mathrm{T}$ Cell Infiltration in Relation to Breast 
Cancer Survival and Molecular Subtypes. Breast Cancer Res Treat (2011) 130:645-55. doi: 10.1007/s10549-011-1647-3

82. Krummel MF, Allison JP. CD28 and CTLA-4 Have Opposing Effects on the Response of T Cells to Stimulation. J Exp Med (1995) 182:459-65. doi: 10.1084/jem.182.2.459

83. Chambers CA, Kuhns MS, Egen JG, Allison JP. CTLA-4-MEDIATED INHIBITION IN REGULATION OF T CELL RESPONSES: Mechanisms and Manipulation in Tumor Immunotherapy. Immunology (2001) 19:56594. doi: 10.1146/annurev.immunol.19.1.565

84. Vonderheide RH, LoRusso PM, Khalil M, Gartner EM, Khaira D, Soulieres D, et al. Tremelimumab in Combination With Exemestane in Patients With Advanced Breast Cancer and Treatment-Associated Modulation of Inducible Costimulator Expression on Patient T Cells. Clin Cancer Res (2010) 16:3485-94. doi: 10.1158/1078-0432.CCR-10-0505

85. McArthur HL, Diab A, Page DB, Yuan J, Solomon SB, Sacchini V, et al. A Pilot Study of Preoperative Single-Dose Ipilimumab and/or Cryoablation in Women With Early-Stage Breast Cancer With Comprehensive Immune Profiling. Clin Cancer Res (2016) 22:5729-37. doi: 10.1158/1078-0432.CCR16-0190

86. Bardhan K, Anagnostou T, Boussiotis VA. The PD1:PD-L1/2 Pathway From Discovery to Clinical Implementation. Front Immunol (2016) 7:550. doi: 10.3389/fimmu.2016.00550

87. Topalian SL, Drake CG, Pardoll DM. Targeting the PD-1/B7-H1(PD-L1) Pathway to Activate Anti-Tumor Immunity. Curr Opin Immunol (2012) 24:207-12. doi: 10.1016/j.coi.2011.12.009

88. Nishino M, Ramaiya NH, Hatabu H, Hodi FS. Monitoring ImmuneCheckpoint Blockade: Response Evaluation and Biomarker Development. Nat Rev Clin Oncol (2017) 14:655-68. doi: 10.1038/nrclinonc.2017.88

89. Mansfield AS, Aubry MC, Moser JC, Harrington SM, Dronca RS, Park SS, et al. Temporal and Spatial Discordance of Programmed Cell Death-Ligand 1 Expression and Lymphocyte Tumor Infiltration Between Paired Primary Lesions and Brain Metastases in Lung Cancer. Ann Oncol (2016) 27:1953-8. doi: 10.1093/annonc/mdw289

90. Strickland KC, Howitt BE, Shukla SA, Rodig S, Ritterhouse LL, Liu JF, et al. Association and Prognostic Significance of BRCA1/2-Mutation Status With Neoantigen Load, Number of Tumor-Infiltrating Lymphocytes and Expression of PD-1/PD-L1 in High Grade Serous Ovarian Cancer. Oncotarget (2016) 7:13587-98. doi: 10.18632/oncotarget.7277

91. Gottlieb CE, Mills AM, Cross JV, Ring KL. Tumor-Associated Macrophage Expression of PD-L1 in Implants of High Grade Serous Ovarian Carcinoma: A Comparison of Matched Primary and Metastatic Tumors. Gynecol Oncol (2017) 144:607-12. doi: 10.1016/j.ygyno.2016.12.021

92. Gupta HB, Clark CA, Yuan B, Sareddy G, Pandeswara S, Padron AS, et al. Tumor Cell-Intrinsic PD-L1 Promotes Tumor-Initiating Cell Generation and Functions in Melanoma and Ovarian Cancer. Signal Transduct Target Ther (2016) 1. doi: 10.1038/sigtrans.2016.30

93. Clark CA, Gupta HB, Curiel TJ. Tumor Cell-Intrinsic CD274/PD-L1: A Novel Metabolic Balancing Act With Clinical Potential. Autophagy (2017) 13:987-8. doi: 10.1080/15548627.2017.1280223

94. Clark CA, Gupta HB, Sareddy G, Pandeswara S, Lao S, Yuan B, et al. TumorIntrinsic PD-L1 Signals Regulate Cell Growth, Pathogenesis, and Autophagy in Ovarian Cancer and Melanoma. Cancer Res (2016) 76:6964-74. doi: 10.1158/0008-5472.CAN-16-0258

95. Thorburn A. A New Mechanism for Autophagy Regulation of Anti-Tumor Immune Responses. Autophagy (2020) 16:2282-4. doi: 10.1080/ 15548627.2020 .1817286

96. Jin J, Zhao Q. Emerging Role of mTOR in Tumor Immune Contexture: Impact on Chemokine-Related Immune Cells Migration. Theranostics (2020) 10:6231-44. doi: 10.7150/thno.45219

97. Shanware NP, Bray K, Eng CH, Wang F, Follettie M, Myers J, et al. Glutamine Deprivation Stimulates mTOR-JNK-Dependent Chemokine Secretion. Nat Commun (2014) 5:4900. doi: 10.1038/ncomms5900

98. Shin SY, Lee DH, Lee J, Choi C, Kim JY, Nam JS, et al. C-C Motif Chemokine Receptor 1 (CCR1) is a Target of the EGF-AKT-mTOR-STAT3 Signaling Axis in Breast Cancer Cells. Oncotarget (2017) 8:94591-605. doi: 10.18632/ oncotarget. 21813

99. Tu X, Qin B, Zhang Y, Zhang C, Kahila M, Nowsheen S, et al. PD-L1 (B7H1) Competes With the RNA Exosome to Regulate the DNA Damage
Response and Can Be Targeted to Sensitize to Radiation or Chemotherapy. Mol Cell (2019) 74:1215-26.e1214. doi: 10.1016/j.molcel.2019.04.005

100. Escors D, Gato-Canas M, Zuazo M, Arasanz H, Garcia-Granda MJ, Vera R, et al. The Intracellular Signalosome of PD-L1 in Cancer Cells. Signal Transduct Target Ther (2018) 3:26. doi: 10.1038/s41392-018-0022-9

101. Chang CH, Qiu J, O'Sullivan D, Buck MD, Noguchi T, Curtis JD, et al. Metabolic Competition in the Tumor Microenvironment Is a Driver of Cancer Progression. Cell (2015) 162:1229-41. doi: 10.1016/j.cell.2015.08.016

102. Keenan TE, Tolaney SM. Role of Immunotherapy in Triple-Negative Breast Cancer. J Natl Compr Canc Netw (2020) 18:479-89. doi: 10.6004/ jnccn.2020.7554

103. Emens LA, Molinero L, Loi S, Rugo HS, Schneeweiss A, Dieras V, et al. Atezolizumab and Nab-Paclitaxel in Advanced Triple-Negative Breast Cancer: Biomarker Evaluation of the IMpassion130 Study. J Natl Cancer Inst (2021). doi: 10.1093/jnci/djab004

104. Nanda R, Chow LQ, Dees EC, Berger R, Gupta S, Geva R, et al. Pembrolizumab in Patients With Advanced Triple-Negative Breast Cancer: Phase Ib KEYNOTE-012 Study. J Clin Oncol (2016) 34:2460-7. doi: 10.1200/JCO.2015.64.8931

105. Adams S, Loi S, Toppmeyer D, Cescon DW, De Laurentiis M, Nanda R, et al. Pembrolizumab Monotherapy for Previously Untreated, PD-L1-Positive, Metastatic Triple-Negative Breast Cancer: Cohort B of the Phase II KEYNOTE-086 Study. Ann Oncol (2019) 30:405-11. doi: 10.1093/annonc/ mdy518

106. Dirix LY, Takacs I, Jerusalem G, Nikolinakos P, Arkenau HT, Forero-Torres A, et al. Avelumab, an Anti-PD-L1 Antibody, in Patients With Locally Advanced or Metastatic Breast Cancer: A Phase 1b JAVELIN Solid Tumor Study. Breast Cancer Res Treat (2018) 167:671-86. doi: 10.1007/s10549-0174537-5

107. Tolaney SM, Kalinsky K, Kaklamani VG, D'Adamo DR, Aktan G, Tsai ML, et al. Eribulin Plus Pembrolizumab in Patients With Metastatic TripleNegative Breast Cancer (ENHANCE 1): A Phase Ib/II Study. Clin Cancer Res (2021). doi: 10.1158/1078-0432.CCR-20-4726

108. Nanda R, Liu MC, Yau C, Shatsky R, Pusztai L, Wallace A, et al. Effect of Pembrolizumab Plus Neoadjuvant Chemotherapy on Pathologic Complete Response in Women With Early-Stage Breast Cancer: An Analysis of the Ongoing Phase 2 Adaptively Randomized I-SPY2 Trial. JAMA Oncol (2020) 6:676-84. doi: 10.1001/jamaoncol.2019.6650

109. Schmid P, Salgado R, Park YH, Munoz-Couselo E, Kim SB, Sohn J, et al. Pembrolizumab Plus Chemotherapy as Neoadjuvant Treatment of HighRisk, Early-Stage Triple-Negative Breast Cancer: Results From the Phase 1b Open-Label, Multicohort KEYNOTE-173 Study. Ann Oncol (2020) 31:56981. doi: 10.1016/j.annonc.2020.01.072

110. Wang J, Sun J, Liu LN, Flies DB, Nie X, Toki M, et al. Siglec-15 as an Immune Suppressor and Potential Target for Normalization Cancer Immunotherapy. Nat Med (2019) 25:656-66. doi: 10.1038/s41591-019-0374-x

111. Brown JS, Sundar R, Lopez J. Combining DNA Damaging Therapeutics With Immunotherapy: More Haste, Less Speed. Br J Cancer (2018) 118:312-24. doi: $10.1038 /$ bjc.2017.376

112. Comprehensive Molecular Portraits of Human Breast Tumours. Nature (2012) 490:61-70. doi: 10.1038/nature11412

113. Samstein RM, Lee CH, Shoushtari AN, Hellmann MD, Shen R, Janjigian YY, et al. Tumor Mutational Load Predicts Survival After Immunotherapy Across Multiple Cancer Types. Nat Genet (2019) 51:202-6. doi: 10.1038/ s41588-018-0312-8

114. Ablasser A, Goldeck M, Cavlar T, Deimling T, Witte G, Röhl I, et al. cGAS Produces a 2'-5'-Linked Cyclic Dinucleotide Second Messenger That Activates STING. Nature (2013) 498:380-4. doi: 10.1038/nature12306

115. Ribas A, Wolchok JD. Cancer Immunotherapy Using Checkpoint Blockade. Science (2018) 359:1350-5. doi: 10.1126/science.aar4060

116. Heijink AM, Talens F, Jae LT, van Gijn SE, Fehrmann RSN, Brummelkamp TR, et al. BRCA2 Deficiency Instigates cGAS-Mediated Inflammatory Signaling and Confers Sensitivity to Tumor Necrosis Factor-AlphaMediated Cytotoxicity. Nat Commun (2019) 10:100. doi: 10.1038/s41467018-07927-y

117. Fridman WH, Zitvogel L, Sautès-Fridman C, Kroemer G. The Immune Contexture in Cancer Prognosis and Treatment. Nat Rev Clin Oncol (2017) 14:717-34. doi: 10.1038/nrclinonc.2017.101 
118. Nolan E, Savas P, Policheni AN, Darcy PK, Vaillant F, Mintoff CP, et al. Combined Immune Checkpoint Blockade as a Therapeutic Strategy for BRCA1-Mutated Breast Cancer. Sci Transl Med (2017) 9:eaal4922. doi: 10.1126/scitranslmed.aal4922

119. Permata TBM, Hagiwara Y, Sato H, Yasuhara T, Oike T, Gondhowiardjo S, et al. Base Excision Repair Regulates PD-L1 Expression in Cancer Cells. Oncogene (2019) 38:4452-66. doi: 10.1038/s41388-019-0733-6

120. Pilié PG, Gay CM, Byers LA, O’Connor MJ, Yap TA. PARP Inhibitors: Extending Benefit Beyond BRCA-Mutant Cancers. Clin Cancer Res (2019) 25:3759-71. doi: 10.1158/1078-0432.CCR-18-0968

121. Stewart RA, Pilié PG, Yap TA. Development of PARP and ImmuneCheckpoint Inhibitor Combinations. Cancer Res (2018) 78:6717-25. doi: 10.1158/0008-5472.CAN-18-2652

122. Shen J, Zhao W, Ju Z, Wang L, Peng Y, Labrie M, et al. PARPi Triggers the STING-Dependent Immune Response and Enhances the Therapeutic Efficacy of Immune Checkpoint Blockade Independent of BRCAness. Cancer Res (2018) 79:canres.1003.2018. doi: 10.1101/318980

123. Pantelidou C, Sonzogni O, De Oliveria Taveira M, Mehta AK, Kothari A, Wang D, et al. PARP Inhibitor Efficacy Depends on CD8(+) T-Cell Recruitment via Intratumoral STING Pathway Activation in BRCADeficient Models of Triple-Negative Breast Cancer. Cancer Discov (2019) 9:722-37. doi: 10.1158/2159-8290.CD-18-1218

124. Yélamos J, Moreno-Lama L, Jimeno J, Ali SO. Immunomodulatory Roles of PARP-1 and PARP-2: Impact on PARP-Centered Cancer Therapies. Cancers (2020) 12:392. doi: 10.3390/cancers12020392

125. Do KT, Manuszak C, Thrash E, Giobbie-Hurder A, Hu J, Kelland S, et al. Immune Modulating Activity of the CHK1 Inhibitor Prexasertib and AntiPD-L1 Antibody LY3300054 in Patients With High-Grade Serous Ovarian Cancer and Other Solid Tumors. Cancer Immunol Immunother (2021). doi: 10.1007/s00262-021-02910-x

126. Zhang Q, Green MD, Lang X, Lazarus J, Parsels JD, Wei S, et al. Inhibition of ATM Increases Interferon Signaling and Sensitizes Pancreatic Cancer to Immune Checkpoint Blockade Therapy. Cancer Res (2019) 79:3940-51. doi: 10.1158/0008-5472.CAN-19-0761

127. Jiao S, Xia W, Yamaguchi H, Wei Y, Chen MK, Hsu JM, et al. PARP Inhibitor Upregulates PD-L1 Expression and Enhances Cancer-Associated Immunosuppression. Clin Cancer Res (2017) 23:3711-20. doi: 10.1158/1078-0432.CCR-16-3215

128. Lee JM, Cimino-Mathews A, Peer CJ, Zimmer A, Lipkowitz S, Annunziata $\mathrm{CM}$, et al. Safety and Clinical Activity of the Programmed Death-Ligand 1 Inhibitor Durvalumab in Combination With Poly (ADP-Ribose) Polymerase Inhibitor Olaparib or Vascular Endothelial Growth Factor Receptor 1-3
Inhibitor Cediranib in Women's Cancers: A Dose-Escalation, Phase I Study. J Clin Oncol (2017) 35:2193-202. doi: 10.1200/JCO.2016.72.1340

129. Lampert EJ, Zimmer A, Padget M, Cimino-Mathews A, Nair JR, Liu Y, et al. Combination of PARP Inhibitor Olaparib, and PD-L1 Inhibitor Durvalumab, in Recurrent Ovarian Cancer: A Proof-Of-Concept Phase II Study. Clin Cancer Res (2020) 26:4268-79. doi: 10.1158/1078-0432.CCR20-0056

130. Vinayak S, Tolaney SM, Schwartzberg L, Mita M, McCann G, Tan AR, et al. Open-Label Clinical Trial of Niraparib Combined With Pembrolizumab for Treatment of Advanced or Metastatic Triple-Negative Breast Cancer. JAMA Oncol (2019) 5:1132-40. doi: 10.1001/jamaoncol.2019.1029

131. Alimzhanov M, Soulard P, Zimmermann A, Schroeder A, Mehr KT, Amendt C, et al. Abstract 2269: ATR Inhibitor M6620 Enhances Anti-Tumor Efficacy of the Combination of the Anti-PD-L1 Antibody Avelumab With PlatinumBased Chemotherapy. Immunology (2019), 2269-9. doi: 10.1158/15387445.AM2019-2269

132. Sun Q, Guo Y, Liu X, Czauderna F, Carr MI, Zenke FT, et al. Therapeutic Implications of P53 Status on Cancer Cell Fate Following Exposure to Ionizing Radiation and the DNA-PK Inhibitor M3814. Mol Cancer Res (2019) 17:2457-68. doi: 10.1158/1541-7786.MCR-19-0362

Conflict of Interest: EY: Advisory Board: Astrazeneca, Bayer, Clovis, Strata Oncology; Research Support to Institution: Eli Lilly, Novartis, Puma Biotechnologies.

The remaining author declares that the research was conducted in the absence of any commercial or financial relationships that could be construed as a potential conflict of interest.

Publisher's Note: All claims expressed in this article are solely those of the authors and do not necessarily represent those of their affiliated organizations, or those of the publisher, the editors and the reviewers. Any product that may be evaluated in this article, or claim that may be made by its manufacturer, is not guaranteed or endorsed by the publisher.

Copyright (C) 2021 Clark and Yang. This is an open-access article distributed under the terms of the Creative Commons Attribution License (CC BY). The use, distribution or reproduction in other forums is permitted, provided the original author(s) and the copyright owner(s) are credited and that the original publication in this journal is cited, in accordance with accepted academic practice. No use, distribution or reproduction is permitted which does not comply with these terms. 